2016,2 (1), 88-109

Recibido:28-04-2016 Aceptado: 17-08-2016

Palabras clav

Evaluaóón nutricional.

Keywords:

Nu rritional evaluation.
Estudiantes de la Maestria en

Nullición y Dietét ica. Enely Llanos, Diana Mesones:Lisbeth Mufioz; Gabriela Peña, Juana Oscorima Rocío Valenzuela.

\section{Evaluación Nutricional de las Promotoras de la Micro Red José Carlos Mariátegui en el Distrito de San Juan de Lurigancho}

Nutritional assessments of promoters of the nzicro nenvork José Manatejui in the district of San Juan de Lurgancho

Llanos Enely; Mesones Diana; Muñoz Lisberh;

Peña Gabriela; Oscorima Juana; Valenzuela Roció.

Estudiantes de la Maestría en Nu crición y Dietética.

Universidad Femen ina del Sagrado Corazón

RESUMEN: Eli presente trabajo fue llevado a cabo con los promotores de salud de la micro red José Carlos Mariátegui de San Juan de Lurigancho. Una entrevista personal y una evaluación nuaicional fueron realizadas a cada uno de los promotores para determinar su actual estado nutriciona.I.

Después que se hallaron los resLulldos, vaiios de ellos estuvieron con sobrepeso y obesidad, ellos fueron capacitados sobre la dieta balanceada.

ABSTRACT: The present work was carried out witb the heaJth promoters of rhe muero network Jose Carlos Mariategui of San Juan $<$ le Lurigancho. A personal inten-iew ans a mutrirional assessment were conducted to each of thc promoters to dererminc their current nutritional sratus.

$J \backslash f t e r$ the rsults were found, severa) of them were ovenveigbt and obese, they were trained on balanced cliet. 


\section{INTROD UCCIÓN}

S

an Juan de Lurigancho es uno de los 43 distritos de la Prm'Íncia de Lima. Está ubicado al Noreste de Lima Metropolitana. Según el INEI (2014), es el distrito con mayor población del Perú, con 1 millón 069 mil 566 residentes'. Esta situación produce una mayor demanda de senricios de educación, alimentación y serYicios básicos por parte de los habitantes los que tienen que ser atendidos por el sector público y privad $o^{2}$.

Segün la Oficina de Epidemiología de Ja Dirección de Salud (DISA), en los úl timos años en San Juan de Lurigancho se vienen incrementad o el número de pacientes que padecen enfermedades crónicas no transmisibles como hiperten sión, diaberes ) obesidad ${ }^{1}$.

La Red de Salud San J uan de Lurigancho del distrito del mismo nombre, actualmente cuenta con 34 establccimien tosde saJud ubicados en zonas estratégicas también cuenta con un hospitaJ, gue es un establecimicnw anitario donde se atiende a los enfermos para proporcionar los diagnósticos y tratamícnro que necesitan¹.

La promoción de Ja salud es una estrategia de la salud pli blica que propone incremen tar oportunidad es para la población a través de la mejora de las condiciones de Yida. Es un proceso que proporciona a las personas los medios necesarios para mejorar su salud y ejercer un mayor comrol sobre la misma. Consiste en un conjunto de acciones encaminadas al desarrollo de condiciones fworables para la salud que pueden darse a ni,el de la escuela, familia, municipio, centros laborales $r$ comunidad, cuyo objetivo es ir disminuyendo las brechas de las inequidades sociales existen tes, que son la fuente principal de problemas de salud pública de nuestra sociedad .

Los promotores de salud desempeñan u n papel clave en la tarea de mejorar la salud. Son considerados como educadores de su comunid ad, es decir, son personas que orien tan y transfieren a la población conocimientos sobre temas de salud r les moci,an a llevar una ,ida más sana. Sin ellos, muchas personas posiblemenre oo recibirían esa bliosa información. Los promotores de salud efecti,ros poseen cualidades especiales: conocen bien a sus comunidades, están dedicadosa mejorar la salud de la comunidad a la que pertenecen, les agrada enseñar a los demás, se sienten cómodos frente a un grupo y saben cómo trabajar con ellos ${ }^{4}$.

Se trata de personas que integran la comunidad en la gue trabajan y que, si bien no forman parte del sistema oficial de salud, cuentan con el apoyo de éste, çue les brinda u na breve formación con el fin de que estén en condiciones de reafü ar su trabajo. De esta manera,el promotor de salud está capacitado para brindar atención primaria de la salud en su comunidad y ser un "puente" entTe ésta y Jos servicios oficiales de salud. En u mayoría, han sido elegidos después de realizar una selección entre otras personas de su comunidad. Tienen la función de fomentar las prácticas adecuadns de cuidado de la salud, con la promoción y el impulso de estas pautas en la comu nidad a la que pertenecen ${ }^{5}$.

El promotor de saJud es visco como el agente que cond uce y enseña la forma de realizar cambios, pa ra lo cual es indispensable que tengan acceso constante a in formación técnica y metodológica que le permita mejorar la calidad de su trabajo. J $\backslash$ simjsmo, existe la expectativa de que los promotores tengan una conducta ejemplar, es decir, se conviertan en un "modelo" para Ja comu nidad . Lamentablemente, la mayoría de personas dedicadas a esca importante $\boldsymbol{r}$ ardua labor no tienen la posibilidad o el tiempo necesario para conocer $\boldsymbol{r}$ atender adecuadamente el estado de su salud.

Por medio deJ presente trabajo se realizó una elTaluación nu uicionaJ integral a los promotores de salud de San Juan de Lurigancho con la cual fue posible realizar el diagnóstico respectivo. También recibieron educación nutricional a través de trípticos sobre alimen rnción saludable y balanceada y sobre 


\section{UNIFÉ -EPG}

Las enfermedades crónicas no transmisibles con los correspondientes consejos y .recomendaciones nu tricionales pata cada una de ellas. De esta manera se contribuye a mejorar la calidad de vjda de los promotores de salud y al mismo tiempo Los conocimientos aprendidos serán transmitidos a sus familias y a los vecinos del distritO.

\section{MÉTODO}

Elgrupo objetivo es denueve (9) Promotores de los Centros de Salud, de la Microred José Carlos I\لfariátegLú -Red de Salud SanJuan dé Luriganého, cuya jurisdicción per tenece al Instituto de Gestión de Servicios de Salud (IGSS); quienes laboi:an voluntariamente para su comunidad y muchas veces descuidan su propia salud.

Lamera que se buscó foe queJas Promotoras conozcan su estado nutricional y también como logtar una buena alimentación, sobre rodo en quienes padecen algunas enfermedadeso dolencias. Con ello se podria torrnlece-r los conocimientos de las Promotoras quienes como agentes de cambio podrían talizar una labor de sensibilización a la comunidad. Para ello se requiere información actualizada y hen:amientls como mpttcps con conceptos importantes y claves para colahorar con la prevención de enfer medades, promoción de l.a salud y alimentación saludable en la comunidad.

Las estudiantes de la Maestría de Nutt:idón y Dietética (14) con el fin de crear conciencia e involucrarse en la solución de 10s pwblemas que afrtct:an a la población en general, realizaron una evaluaciónantropometríca ydietél;ica alasPromoto.ras, para así determinar su dJagnóstico nutriciooal, rambjén brindaron educación nvtticional sobre alimentación balanceada e información específica acorde a las enfermedades o:ónicas no transmisibles pre- existentes en esta dichas promotoras, para lo cual eplea ron trípticos específicos por enfermedad frecuente con datos de una cor.tecta alimentación: Diabetes Mellitus tipo II, Hipertensión Arterial, Hipercolesterolemia ${ }_{1}$ Hipertrigliceridemia, Obesidad y Gastritis; finalmente, los ejemplares de los trípticos utilizados fueron entregados a cada Promotora participante para facilitar su tol de voluntarias ert la comno.idad $y$ con ello puedan compartir dichos conocimientos adquiridos.

I?.1objetivo general fue brindar el diagnóstico nutricional a las promotoras de salud mediante la aplicación de la evaluación nutricional integral e li:npartitconodmieotos puntuales sobre enfermedades no rra.usrn:isibles y alimentación balanceada.

El primer objetivo específico foe mostrarles objetivamen te a las Ptol'notoras de salud, tómo se encuentra su Bstado Nu tiicional y sensibiliza.das pru:a que mejoren su alimentación evitando Jas complicacjones de sus enfermedades crónicas actuales. El s:egundo objetivo e!>pecífico fue guelas P.romqtoras de Salü:d pro1lluevan la alimentación saludable $y$ balanceada en sus familias y en Ja comunidad a través de sus actividades propins como pane de su voluntariado.

Las Actividades se dividieron en tres fases: antes, durante y después del u--abajo de campo:

\section{- Antes;}

1. Elaboración de los mensaies nuti-ic onales pra cada tipo de enfermedad, relrisado por todas Jas ,nu.tricionistas.

3. Cteatjón de trípticos con la información de orientaci(>n para las enfermedades mas (recuentes y ali mentación saludable.

4. Validación de los trípticos.

5. Diseño de un flujograma de actividades para el trabajo de. campo.

\section{- Durante:}

L Cada estudiante tuvo tma promotora para realizar el trabajo de cam po.

2. Cada ptomotota fue evaluada.

Antropometría (sele medió la circunferencia de cintura. se le pesó y talló para determinar el IMC).

Clínicos (se le preguntó sobre los síntomas y signos gue han e\$tado presentando en 10\$ últimos 3 meses) y

Dietéticos (se les aplicó la Encuesta de Recordatorio de 24 hmas).

3. El regístte.> de la Evaluac.ión nutriciona) fue 
en un formato ad hoc (Anexo 02).

4. Dependiendo de la enfermedad que refiriera la Promotora se le brindó la Educación nurricional con apoyo del tríptico correspondiente (Anexos del 03 al 09) y la información obtenida en la Evaluación nutricional integral, enfatizando que toda enfermedad es manejable siendo importante que se evite las complicaciones.

5. Se le explicó sobre la Alimentación saludable y balanceada con apoyo del tríptico (Anexo 09) y se le reforzó en la promoción de esta alimentación como parte de los estilos de vida saludable que incluye el ejercicio.

6. Finalmente, se le dio un juego de trípticos para que lo empleen cuando desarrollen sus actividades con la comunidad.

\section{- Después:}

1. Sistematizar el trabajo de campo

2. Evaluar la asimilación de los mensajes por parte de las Promotoras, y sobre todo si las estudiantes se dejaron entender.

3. Ingresar los datos en una base de datos creada para este .fin en el programa Excel 2013 y realizar el control de calidad de los datos.

4. Analizar los datos mediante la estadística descriptiva en el mismo programa Excel 2013 (porcentaje, frecuencias y Figuras).

\section{RESULTADOS Y DISCUSIÓN}

\section{Índice de masa corporal y edad}

Se relacionó el cliagnóstico de IlvlC obtenido con los rangos de edad de los nueve (9) promotores de salud.

Figura 1 Diagnóstico del IMC según edad 3

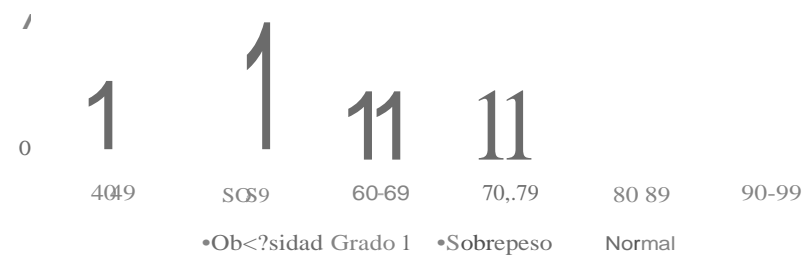

En la figura se puede apreciar que 33.33\% (3) de los promotores de salud evaluados presen taron obesidad grado I, 44.44 $\mathrm{Vo}$ (4) de ellos se cliagnosticaron con sobrepeso y $22.22 \%$ (2) de ellos tuvieron un IMC normal. La obesidad grado I estuvo presente en los rangos de edades de 40-49 en $11.11 \%$ (1 promotor), de 60 a 69 años en $11.11 \%$ ( 1 promotor) y de 70 a 79 años en $11.11 \%$ (1 promotor). El sobrepeso se presen tó en los rangos de edades de 50-59 años en 22.22\% (2 promotores), de 60-69 años en 11.11\% (1 promotor) y de 70 a 79 años en $11.11 \%$ (1 promotor).

\section{Índice de masa corporal y sexo}

Se evaluó el cliagnóstico del IMC en relación al sexo obteniéndose los siguientes resultados:

Fígura 2. Diagnóstico del IMC según sexo

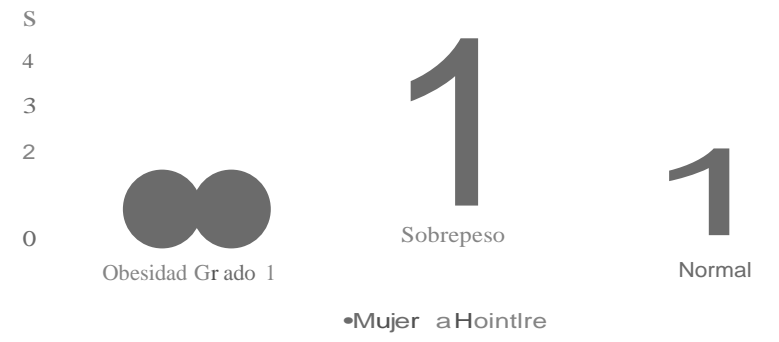

En la Figura 2 se observa que de los nueve (9) promotores evaluados el $77.78 \%$ (1) son mujeres y el 22.22\% (2) son hombres. En lo gue respecta a los criterios de cliagnóstico del IMC, los dos hombres evaluados (100\%) presentaron obesidad grado J. JYiientras que las mujeres evaluadas, una promotora (14.29\%) presentó obesidad grado I, 4 de ellas (57.14\%) fueron cliagnosticadas con sobrepeso y 2 de ellas (28.57\%) tuvieron un IMC normal.

\section{Circunferencia de la cintura y sexo}

Según los resultados obten.idos, en Ja categoria de riesgo carcliovascular moderado hay cuatro mujeres y un hombi::e, quienes representan el 55.56\% del total y en la categoría de riesgo cardiovascular alto hay tres mujeres $y$ 


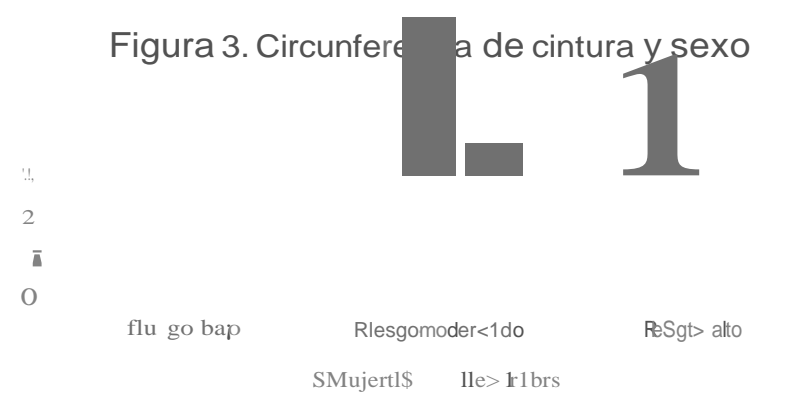

un hom bre quienes representan el $44.44 \%$. Por otro lad0, ninguno de !os promowres presentó una circunferencia de dntura que representara un riesgo carcliovascular bajo.

\section{Grado de instrucción e Índice de Masa Corporal (IMC)}

Figura 4. Grado de Instruccion e IMC

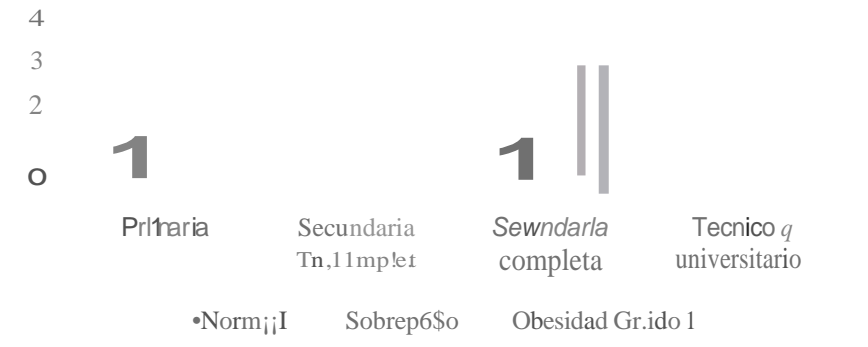

Según la información recolectada sobre eJ grado de instrucción, u.no de los promotores (11.11\%) ha estudiado hasta primaria completa, uno (11.11\%) ha estudiado la secundaria incompléta, 5 (55.56\%) han estudiado la secundaria completa y 2 (22.22\%ii) tienen estudios técnicos o universitarios. Se puede apreciar que las 3 personas (33.33Yo) que tienen obesidad grado I han estudido hasta secundaria completa.

\section{Enfermedades referidas por los promotores evaluados}

El estudio determinó gue las 'enfermedades no trnnsmísibles más prevalentes presentadas por los promotores de salud evaluados fue:roCJ la diabetes mellims tipo 2 en un 33.33\% (3) y la hipertensión arterial también en un 33.33\% (3) de ellos. Esras enfe,rmedades es.tán directamente relacionadas
Figura S. Enfermedades referidas por

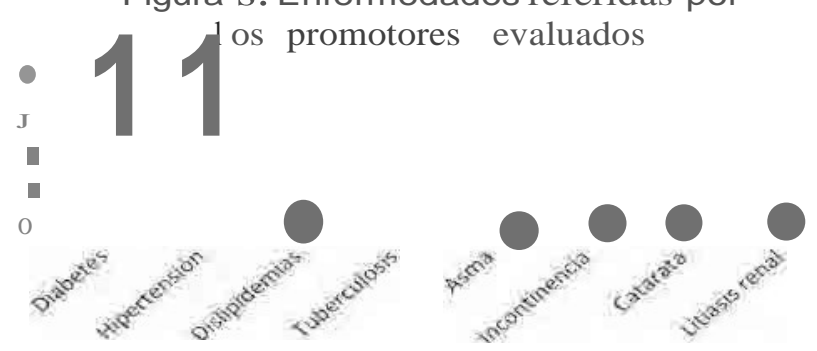

al sobrepeso y La obe idad. Por orto lado, en la Figura $N^{\circ} S$ también se evidencia que existen enfei:nledades pre-existentes, las cuales_hn tenido que ser consjde.rados al momento de la educación nutricional.

\section{Promotores evaluados con conocimiento de enfermedad}

Figura 6. PrQmotores con conocimiento de enfennedad y pmmotores sanos

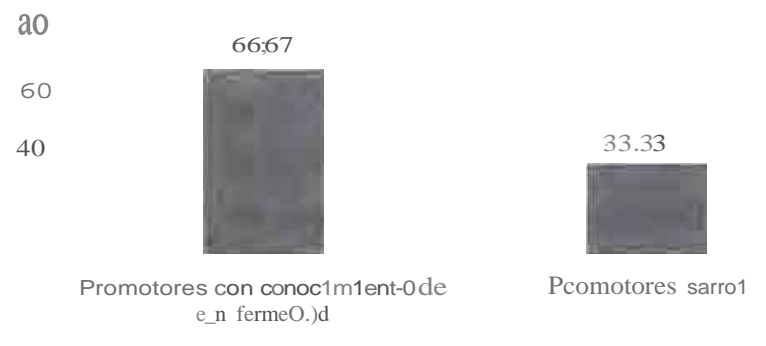

De los prom0tores evaluados, 66.67\% (6) refirieron conocer el padecimiento de algun enfermedad al momentQ de la evaluación, mientras que el $33.33 \%$ (3) del total, se encuentran aparentemente sanos o refieren no padecer -de alguna enfermedad.

\section{Hábitos de los promotores de salud}

Figurq 7. Hábit9s de los pr.omotores -de salud

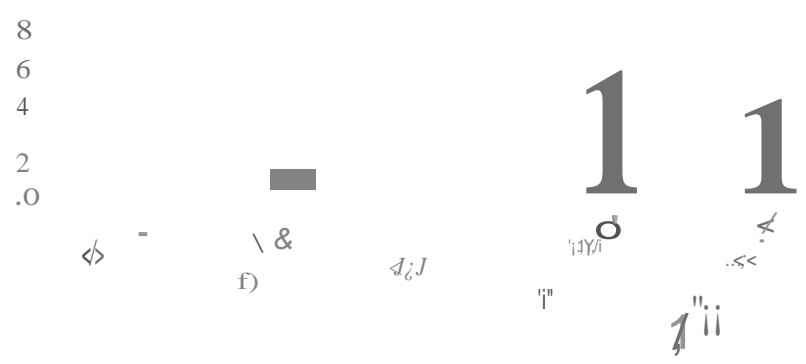


Figura 8.Actividad física/ejercicio

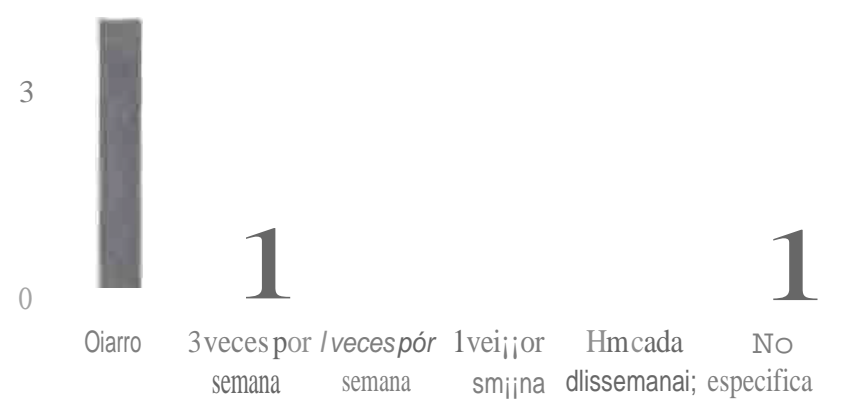

Figura 9. Consumo de

Frituras/snacks/alirnentos azuca rados/gaseosas

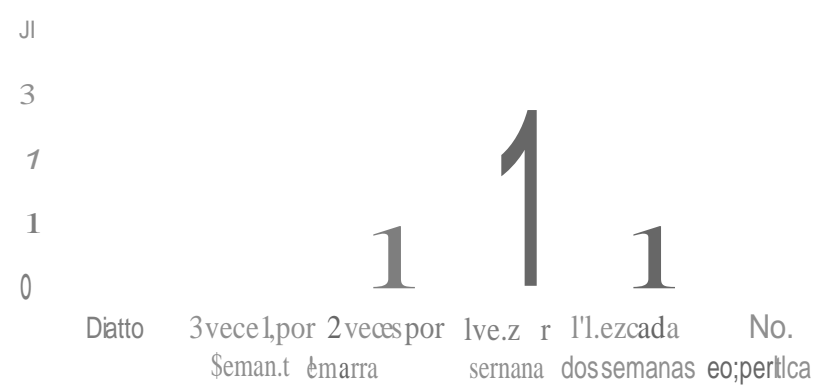

En la Figura 7 se especilican los principales hábitos de los promorores de salud. Entre todos ellos los más CtJmunes fueron la a.cmidad ffsica/ ejercicio, el cual es realizado por 6 de Jos 9 promotores (66.67\%) y el consumo de frimras/ snack/alimen ms a7.ucarados/gaseosas el cual está presente en 5 de los 9 promotores (55.56\%). Sólo uno de ellos (11.11\%) manifesró el consümo de licor en raras ocasiones. Ea la Figura 8 se detalla la frecuencia ele la realización de acthidad física/ ejercicio; se puede apreciar que 4 de ellos (44.44\%) lo realizan diariamente, 1 de eJos (1L.11\%) 2 veces por semana y 1 de ellos responcli6 afirmativamente sóbr:e la práctica de ejru:cicio pero no especificó la frecuencia. En cuanto al consumo de frituras/ snacks/alímentos azucaraJos/gnseosas (Grafico 9), un promotor (11.11\%) lo i-caliza 2 veces a la semana, 3 de ellos (33.33\%) una vez por; semaila y uno de ellos (11.119t)) una vez cada dos semanas.
Frecuencia de consumo y recordatorio de 24 horas

Figura 10

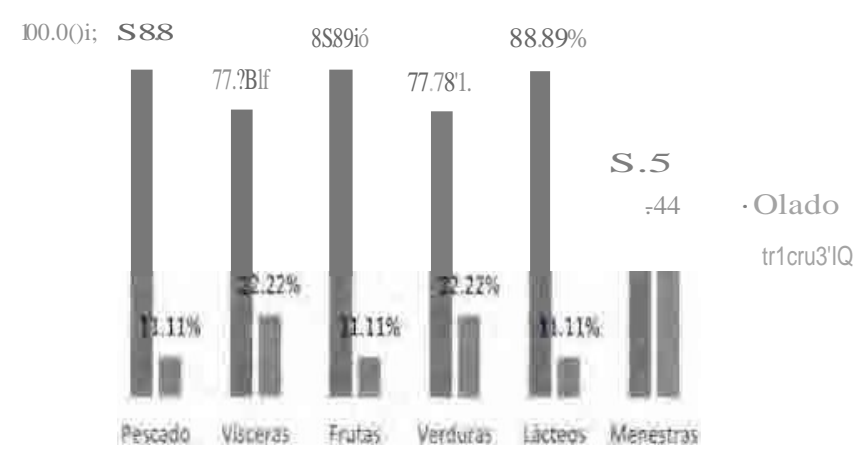

Figura 11. Recordatorio de 24 horas

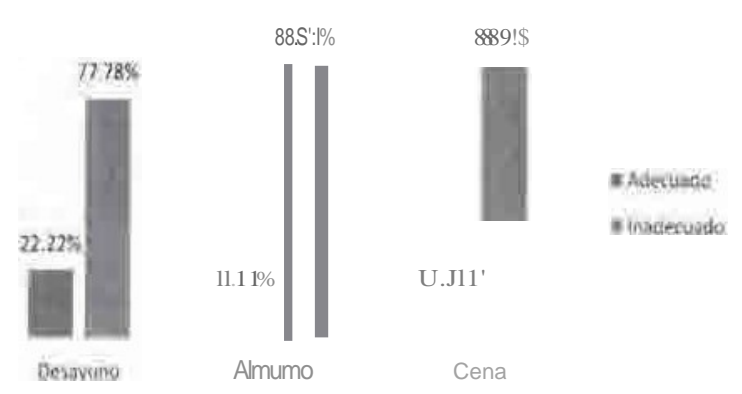

Al enfremar los resulmdo de Frecuencia de Consumo de Alimentos y el Recordatnáo <le 24 Horas (Pigttras 10 y 11 rc:spectivame.nte) en las promotorns evaluadas, podemos decir que no es coherente, pues, al tener un conc; um o de alimentos importantes calificados como Adecuados, esm no se vea reflejado en el consumo de hace 24 b.oras atrás de la entrevista. :bfenos del SOYji, de las Promoo.ras evaluadas muestra un consume> adecuado de las 3 comiJas. principales del elfo.. Podem os concluir que e1 sesgo más evidente es que hayan respondjdo con muy poco acercamien to a Ja verdad en el cuestionario de frecuencia de consumo ele alimentos. Sin embargo, también hay que recalcar que, el formato de Recordatorio de 24 horas no es un instrumento de alta confiabilidad. Como eo toda evaluación, al u tilizar lsre formato no confiable (cuando se utiliza ruslado), necesita Qtm instrwnen to de apoyo, en este caso, el formato de frecuencia de consumo de alimentos. 
Figura 12. kcal consumidas diariamente

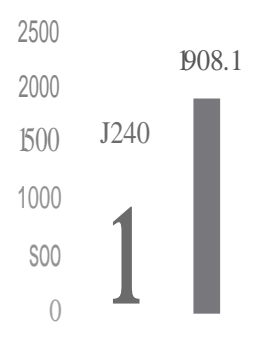

1490

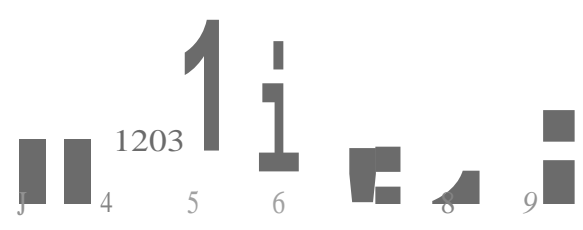

Figura 13. \% kcal consumidas como proteína

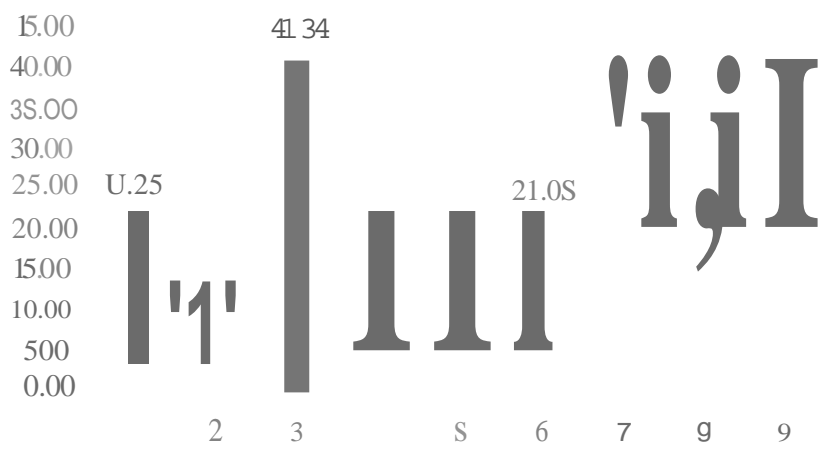

Por teoría sabemos que en las edades post climaterio, requieren un consumo de $1600 \mathrm{kcal}$ en promedio. Según los rcsu.ltad o\$ presentad os en la Figura 12, las pmm otoras evaltiadas consumen diariamente alrededor de 1382.?kcal en promedio, es deór, están por debajo de su requerjmiento diado normal. En cuanco al consu mo de p.roteínas (Pigura 13), este se considera normal, pues van hasta un promedio dt 18.7911 de las calorías totales, considerando ciue según teoria, el requerimiento diario puede oscilar en tre un 10 a $15 \%<1$. AJ enfrentar ambos consumos primótdiales, podernos decir gue es escaso el consomo calórico, pero a su vez $_{1}$ denrro de lo posible, el consumo proteico va acorde, Este es un tema al cual se debe poner especial acención, pues, sabemos que luego de los 50 años Lamasa muscular va decayendo en ambos géneros, )' debemos e $\backslash$ irar la aparición de sa.r:copenia, la cual es muy dificil de remitir.
Comparación de la asimilación de mensaje s sobre la Alimentación Saludable y Balanceada antes y después de la educación nutricional

Figura 14.
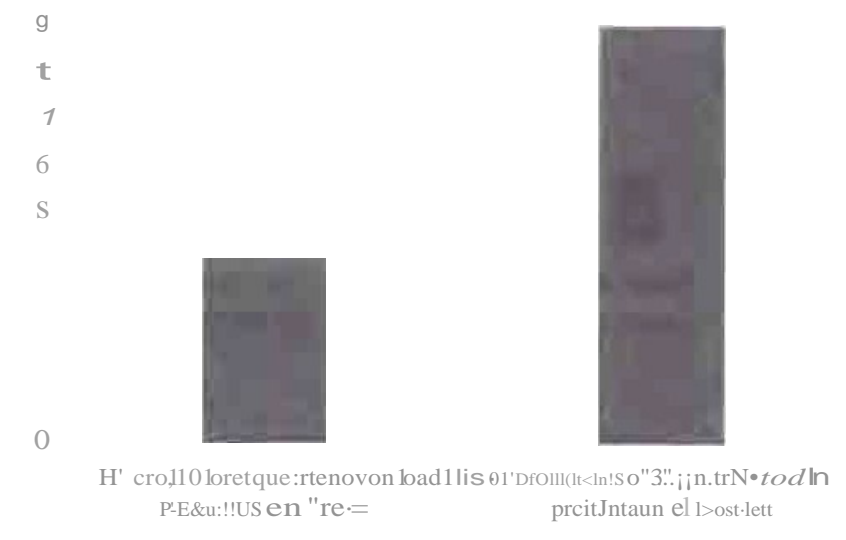

En la Figura 14 se puede observar que al reaijzar el Pre-Test solo 4 de los promotores (44.44\%) respondieron bien todas las preguntas sobre alimentción saludable y balanceada. Después de recibir la educación nutricional, codos los promorores (9), es decir el 100 V > <le ellos respondieron correctamente a las preguntas realizadas, lo gue signJfíca gue los conocimientos ciue recibieron por parte de las estudiantes fue.ron totalmente asimilados, lo que les permitiL1. a:ansmitirlos de maneta adecuada a su comunidad y a sus familias.

\section{CONSIDERACIONES FINALES}

Los servicios de salud que actualmente se brindan a la pobfacjón escán enmarcadüs básicamente en la atención integral con én fasis en pre\Tención y promoción de la salud, con participación ciudadaoá y multisectoriaJ para el logro de comunidades $\mathrm{V}$ ento.rnos 5aJudables` doode muchos de los temas engloban temas de Nutrición y Alimen tación saludable: con la finalidad de prevenir en riesgo de tas enfermedades no transmisib les. Por todo lo antes menc:ionado, La Escuela de Postgtado de la U.niYersida.d Femenina del Sagrado Corazón (Unifê) p.resent't'I mediaJ)te e1 programa de Maestría en Nutrición y 
Dietética con mención en Ciencias de la N utrición y Alimentación Humana, el curso de Taller de Desarrollo de Proyectos en N utrición, a través del cual se desarrollan conocimientos, habilidades, y destrezas en comunidades / centros de salud con la finalidad de diseñar y desarrollar planes y programas que contri buyan a resolver problemas nutricios específicos, a fin de elevar la calidad de vida de la población, en un marco de cuidado y preservación del medio ambiente; en la búsgueda continua de la calidad y, con apego a valores éticos, humanistas y de responsabilidad social.

Este grupo de Promotores de Salud maneja un tipo de información básica sobre la Alimentación saludable y balanceada, indicando muchas de ellas que el factor económico no les permitía variar su alimentación. Asimismo no manejan recomendaciones básicas en cuanto a la alimentación y dieta para cada tipo de enfermedad crónica no transmisible frecuente entre personas adultas, por ello rnientras se les expli.caba la importancia de cumplir las recomendacion es sin la necesidad de variar su dieta, ponían mucho interés viendo gue no se trataba de gastar más sino modificar y balancear su alimentación de acuerdo a las enfermedades que ya presentan.

Por otra parte, los cornetarios finales de las estudiantes fueron los siguientes:

Como participaci.ón individual:

);o- Cumplimos con las expectativas, brindamos información básica, comprensible y fácil de aplicar.

)> Brindamos más confianza a los Promotores para que puedan capacitar no sólo en la alimentación de personas sanas, sino también en la alimentación de personas con enfermedades crónicas no transmisibles; del mismo modo se les enseñó a determinar el IMC en adultos y así ellas lo puedan hacer en su comunidad.

);o- Entre todas las compañeras tuvimos una buena comunicación y cumplimos el objetivo de evaluar, diagnosticar y educar a las Promotoras de manera individual

);0- Al ser profesionales de N utrición las que dimos las consejerías y evaluaciones, pudimos disipar las dudas de las Promotoras, al mismo tiempo que se les facilitó más información mediante los Trípticos que se les entregó sobre las enfermedades no transmisibles más frecuentes y sobre alimentación balanceada, los cuales estaban validados previamen te.

\section{Como participación grupal}

$>$ Los equipos de trabajo inicialm ente se formaron por afinidad; sin embargo, en el transcurso del Trabajo de campo se desintegraron y reagruparon según la necesidad.

$>$ Tuvimos todo el material necesario para los 09 Promotores convocados.

);o- Hubo bastante orden a pesar de que se compartían los mismos espacios, a la vez, no se generó malestar a los Promotores.

Apoyo de la Micro red José Carlos Mariátegui.

);o- Nos brindaron los ambientes necesarios en el momen to para la comodidad delos Promotores y de las estudiantes.

);o- Todos los trabajadores fueron amables y no escatimaron esfuerzos para apoyar el trabajo facilitando la logística.

$>$ Los Promotores de Salud se fueron agradecidos por enriquecer más su información y despejar sus dudas.

> Todos nos apoyaron en el trabajo, fueron muy amables al brindar información detallada.

\section{CONCLUSIONES}

Las estudiantes de Ivlaestría en Nutrición de laUniversidad Femenina del Sagrado Corazón de Jesús (Unifé) del Ilciclo, han podido constatar la relevancia del estado nutricional y la educación alimentaria en promotores(as) de la Micro Red de Salud José Carlos Mariátegui del distrito de San Juan de Lllrigancho . 
Entre los resultados más relevantes se encontraron que el diagnóstico nutricional predominante en los promotores(as) de la Micro Red de Salud José Carlos Mariátegui del distrito de San Juan de Lurigancho es el SOBREPESO en un $44 \%$ (4) y OBESIDAD GRADO Ien un 33\% (3) del total de evaluados. Asimismo, del 44\% (4) de promotores(as) de la Micto Red de Salud José Carlos Ma1átegui del distrito de San Juan de Lurigancho diagnosticados con Sobrepeso, el 50\% (2) de estos se encuentra en el rango de edad de 50 a 59 años.

Se halló que las mujeres son las mas afectadas, presentando riesgo moderado de enfermedades cardiovasculares al evaluarse circunferencia de la cintura. Entre las enfermedades pre- existen tes al momento de la evaluación encontramos que el 66\% de estos refieren padecer de Djabetes Mellitus tipo 2 o de Hipertensión arterial por lo que la educación allmentaria jugó un papel importante.

Por otro lado, se obtuvo que del total de los promotores de la Micro Red de Salud José Carlos Mariátegw del distrito de San Juan de Lurigancho, el 56\% cuenta con estudios secundarios completos, de estos el 60\%, (1 presen ta un diagnóstico de Obesidad Grado I.La totalidad de los promotores con un grado técnico o uruversitario presentaron Sobrepeso y tras la pregunta sobre sus hábitos alimentarios, eJ 55,56\% presenta un consumo de frituras/ snacks/alimentos azucarados/gaseosas entre una y dos veces a Ja semana.

Es por todo lo antes dicho, que la educación alimentaria sobre enfermedades no transmisibles que se brindó a los promotores de salud consistió en ser específica, con mensajes claros y oportunos para la prevención e intervención inmediata y de esta manera sean agentes de cambio mejor preparados.

Contradictoriamente también se evidenció quela carga calórica c:Liaria enpromedio comprendía entre $1382.7 \mathrm{kcal}$, la aual es menor a la necesaria, por lo ciue presentamos dicha información como un sesgo, ya que el reporte de IMC presenta resultados de Sobrepeso y Obesidad.
Al final del trabajo de campo se pudo evidenciar que el $100 \%$ de los promotores (as) de la Micro Red de Salud José Carlos Mariátegui han podido obtener nuevos conocimientos, los cuales fueron identificados al realizar una prueba depre y post-test. En conclusión, se cumplió con la meta y los objetivos planteados para el Trabajo de campo, tan to los Promotores(as) como las estudiantes de maestría de la Unifé quedamos satisfechos con la intervención, además se pudo constatar que es muy importante replicar trabajos como estos en otros puestos $\mathrm{y} / \mathrm{o}$ centros de salud.

\section{REFERENCIAS}

INEI: Lima cuent.a con 9 millones 752 mil habitantes. La República 2015 Enero 17; Sec. Sociedad. Disponible en: http://larepublica.pe/17-01-2015/ inei-lima-tiene-9-millones-752-mil-habirantes

Municipalidad Distrital de SanJuan de Lurigancho. Plan de Desarrollo Concertado 20152021. Disponible en: http://munisjl.gob. pe / tr a nsparencia / plan-de-desarrollo concertado-2015-2021.pdf

GLúa de Gestión de la Estrategia de Escuelas Promotoras de Salud. Nlinisterio de Salud. Dirección General de Promoción de la Salud. Ministerio de Educación. Lima: Ministerio de Salud; 2006. Disponible en: ftp: / / ftp2.minsa .gob.pe/descargas /dgps / docwnentos/guiadegestion.pdf

Su corazón, su vida: Manual del promotor y promotora de salud. U.S. Department Of Health And Hu man Services, Nacional InstituteS of Health, Nacional Heart, Lung and Blood Institu te. 2008. Disponible en: h ttp: / /www.nhl bi.nih.gov / files / docs / resources/heart/lat mnl sp.pdf

Promotor de salud. Trabajo y motivaciones. Buen Inicio, UN ICEF/ PERU-USAID. Lima, 2003. Disponible en: xa.yimg.com/ kq/groups / 28134856 / 665514084/ naine/

\section{PromotorSalud.pdf}




\section{ANEXOS}

ANEXO 1.

FOTOS DEL TRABAJO DECAtllPO
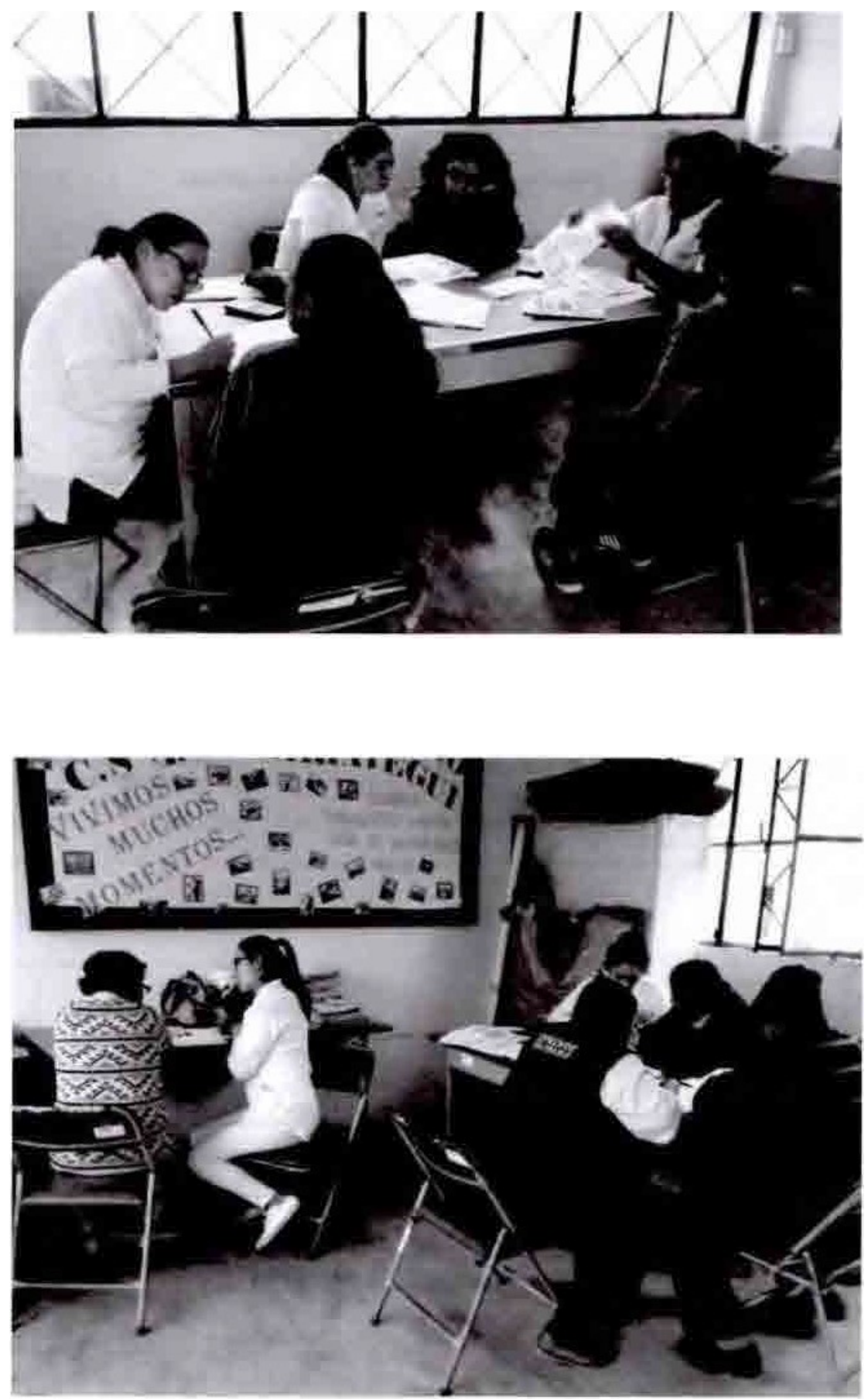


\section{UNIFÉ - EPG}

\section{ANEXO 2. FORMATO DE RECOLECCIÓN DE DATOS DEL TRABAJO DE CAMPO.}

I. DATOS GENERALES

Nombres y Apellidos

11 de la promotora:

Fecha de

12.- Nacimiento:

¿Cuántos años vive

1.4.- en Lima?

Número de

1.6.- hijos:

1.8.- Religión:

110.- Grado de instrucción:

secundaria completa
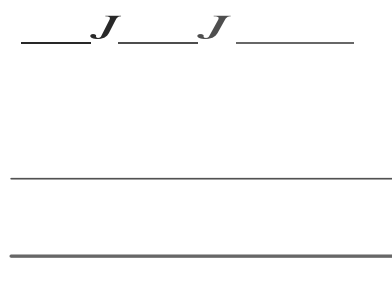

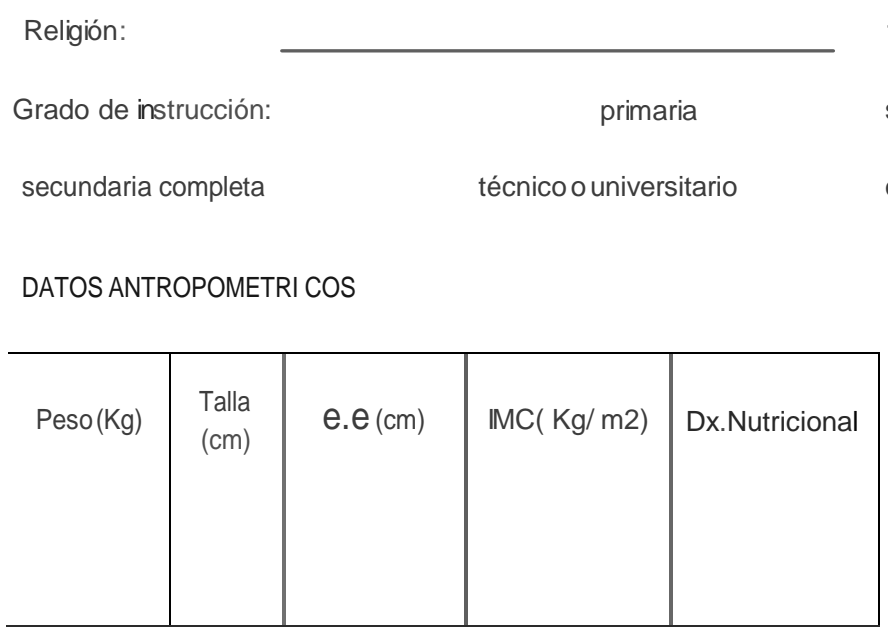

11.

DATOS ANTROPOMETRI COS

Criterios de diagnóstico de IMC(Kg/m2)-OMS

\begin{tabular}{|c|c|c|c|c|}
\hline Normal & $\begin{array}{c}\text { Sobrep } \\
\text { eso }\end{array}$ & $\begin{array}{c}\text { Ob.Grado1 } \\
\text { (leve) }\end{array}$ & $\begin{array}{c}\text { Ob.Grado 11 } \\
\text { (moderada) }\end{array}$ & $\begin{array}{c}\text { Ob. Grado } \\
111 \text { (severa) }\end{array}$ \\
\hline $\mathbf{1 8 . 5 - 2 4 . 9}$ & $25-29.9$ & $30-34.9$ & $35-39.9$ & 40 \\
\hline
\end{tabular}

111

\begin{tabular}{|l|c|c|}
\hline Exámenes & $\begin{array}{c}\text { Resulta } \\
\text { dos }\end{array}$ & $\begin{array}{c}\text { Valores } \\
\text { Normales- ADA- } \\
\text { NECP }\end{array}$ \\
\hline $\begin{array}{l}\text { Colesterol } \\
\text { Total }\end{array}$ & $<200 \mathrm{mg} / \mathrm{dl}$ \\
\hline $\begin{array}{l}\text { Colesterol } \\
\text { LDL }\end{array}$ & $<130 \mathrm{mg} / \mathrm{dl}$ \\
\hline $\begin{array}{l}\text { Triglicérido } \\
\text { S (ayunas) }\end{array}$ & $<150 \mathrm{mg} / \mathrm{dl}$ \\
\hline \multicolumn{2}{|c|}{} & $<5 \mathrm{mg} / \mathrm{dl}$ \\
\hline
\end{tabular}

DATOS BIOQUÍMICOS
$\mathrm{FECHA}$

13.- ugar de nacimiento

1.5.- Cuántos años vive en San Juande Lurigancho?

1.7.- Edades de hijos:

1.9.-Menopausia

No (

$\operatorname{Si}($

secundaria incompleta

otro

\begin{tabular}{|c|c|c|}
\hline \multicolumn{3}{|c|}{$\begin{array}{l}\text { Riesgo cardiovascular - } \mathbb{C} \text { cunferencia de } \\
\text { Cintura (C.C) ADA }\end{array}$} \\
\hline Normal & $\begin{array}{l}\text { Riesgo } \\
\text { elevado }\end{array}$ & $\begin{array}{c}\text { Riesgo muy } \\
\text { elevado }\end{array}$ \\
\hline$<82 \mathrm{~cm}$ & $82-88 \mathrm{~cm}$ & $>88 \mathrm{~cm}$ \\
\hline
\end{tabular}


IV. DATOS CLINICOS

Marcar con un aspa

(x) ycomplete

Antecedentes

Familiares

Presente

Ausente (

Enfermedades Presente

Ausente (

\begin{tabular}{|l|l|l|l|}
\hline $\begin{array}{c}\text { Enfermeda } \\
\text { des }\end{array}$ & Si & No & Parentesco \\
\hline Diabetes & & & \\
\hline $\begin{array}{l}\text { Hlpertensió } \\
\mathrm{n}\end{array}$ & & & \\
\hline Cáncer & & & \\
\hline Otro: & & & \\
\hline
\end{tabular}

\begin{tabular}{|l|l|l|l|}
\hline $\begin{array}{c}\text { Enfermeda } \\
\text { des }\end{array}$ & Si & No & Medicamentos \\
\hline Diabetes & & & \\
\hline $\begin{array}{l}\text { Hipertensió } \\
\mathbf{n}\end{array}$ & & & \\
\hline $\begin{array}{l}\text { Dislipidemi } \\
\text { as }\end{array}$ & & & \\
\hline Otro: & & & \\
\hline
\end{tabular}

Otros en los últimos 3

meses:

\section{Trastornos}

Gastrointestina

les

\begin{tabular}{|l|l|l|l|}
\cline { 2 - 4 } $\begin{array}{c}\text { Enfermeda } \\
\text { des }\end{array}$ & Si & No & frecuencia \\
\hline Diarreas & & & \\
\hline $\begin{array}{l}\text { Estreñimie } \\
\text { nto }\end{array}$ & & & \\
\hline Alergias $\left(^{*}\right)$ & & & \\
\hline Gases & & & \\
\hline Otro: & & & \\
& & & \\
& & & \\
\hline
\end{tabular}

\section{Hábitos}

\begin{tabular}{l|l|l|l|}
\hline \multicolumn{1}{c|}{ Hábitos } & Si & No & frecuencia \\
\hline $\begin{array}{l}\text { Fumar } \\
\text { cigarrillo }\end{array}$ & & & \\
\hline Beber licor & & & \\
\hline & & & \\
Drogas & & & \\
\hline Actividad Fisica & & & \\
/ Ejercicio & & & \\
\hline $\begin{array}{l}\text { Frturas / } \\
\text { snacks/ } \\
\text { alimentos } \\
\text { azúcarados/ } \\
\text { gaseosas }\end{array}$ & & & \\
\hline
\end{tabular}

\section{DATOS DIETÉTICOS}

$$
\begin{aligned}
& \text { PRE- } \\
& \text { TEST }
\end{aligned}
$$

5.1.- ¿Conoce cuáles la importancia de comer pescado de carnes oscuras?

5.2- ¿conoce elsignificado deallmentacion balanceada?

5.3. ¿Conoce cuanta cantidad de agua es lo recomendado en tomar?

5.4. ¿Conoce cuánto ejercicio se recomienda diariamente?

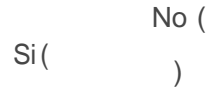

Especifique:

Sil No ( Especifique:

$\mathrm{Si}(\quad)$ No ( specifique:

Si( ) No ( ) Especifique: 
Frecuencia de

consumo

Marcar con un aspa (x) y complete

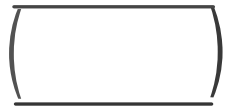

\begin{tabular}{|c|c|c|c|c|c|}
\hline \multirow[b]{2}{*}{ Allmentos } & \multicolumn{5}{|c|}{ Frecuencia } \\
\hline & diario & Interdiario & a veces & nunca & $\begin{array}{l}\text { otra: } \\
\text { espee } \\
\text { flque }\end{array}$ \\
\hline pescado & & & & & \\
\hline vísceras & & & & & \\
\hline frutas & & & & & \\
\hline $\begin{array}{c}\text { verduras } \\
\text { (ensaladas) }\end{array}$ & & & & & \\
\hline ácteos & & & & & \\
\hline menestras & & & & & \\
\hline
\end{tabular}

Recordatorio de 24

horas

motivo:

5.6. El día de ayer su alimentación fue usual?
Si (
No (

5.7 ¿Para cuántas personas prepara el desayuno?

5.9. ¿Para cuántas personas prepara el lonche?

5.8. ¿Para cuántas personas

prepara elalmuerzo?

S.10. ¿Para cuántas personas

prepara lacena?

\begin{tabular}{|c|c|c|c|c|c|c|c|}
\hline $\begin{array}{l}\text { Tiempo } \\
\text { de comida }\end{array}$ & Hora & preparación & Ingredientes & $\begin{array}{l}\text { medidas } \\
\text { caseras }\end{array}$ & $\begin{array}{l}\text { medida en gr } \\
\text { o ce }\end{array}$ & $\begin{array}{l}\text { Energía } \\
\text { (Kcal) }\end{array}$ & prot (gr) \\
\hline
\end{tabular}


Alétheia 2016,3(1) 101

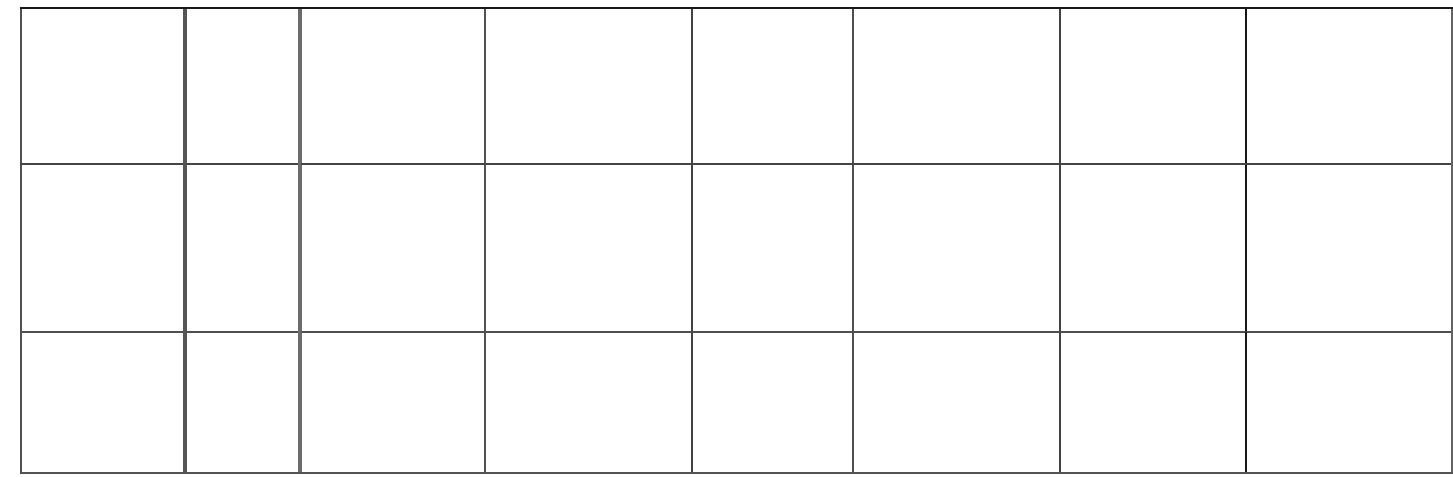

APORTE CALÓRICO

PROMEDIO (Kcal)

POST-

TEST

5.11.- ¿Conoce cuál es la importancia de comer pescado de carnesoscuras?

5.12-¿Conoce el significado de alimentación balanceada?

5.13. ¿Conoce cuanta cantidad de agua es lo recomendado en tomar?

5.14. ¿Conoce cuánto ejercicio se recomienda

diariamente?
$\operatorname{Si}($

Sí(

$\mathrm{SI}($

$\operatorname{Si}($
No (

Especifique;

Especifique:

No (

Especifique:

No (
No (
Especifique:

OBSERVACIONES: 

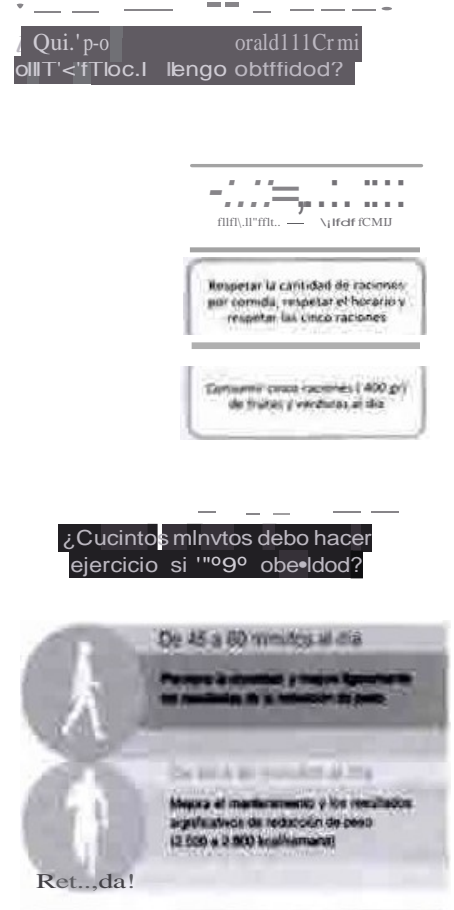

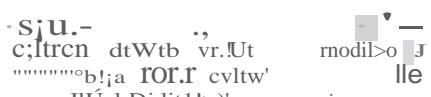
$1>)^{\prime}-$
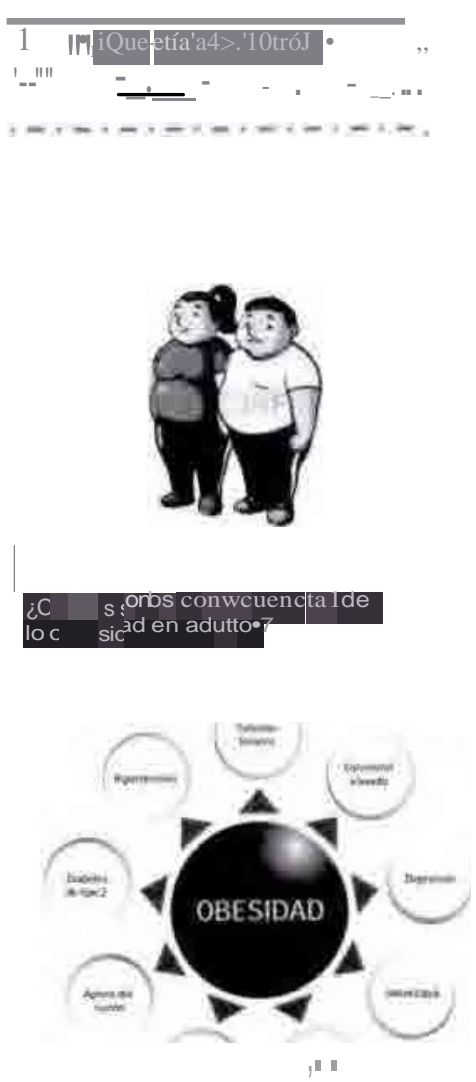

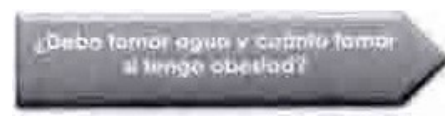

$$
\text { 는 }
$$
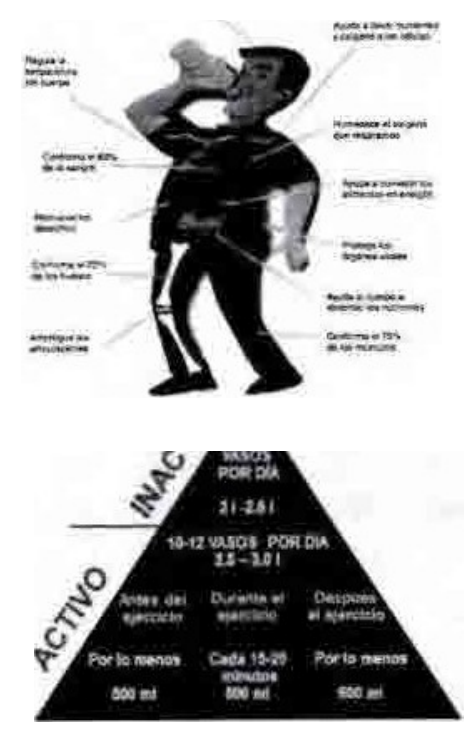

Excuria de Ront grada UNift

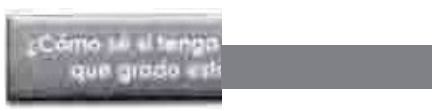

$I M C=\underset{\text { ALIVNA }}{\text { PESO }}$
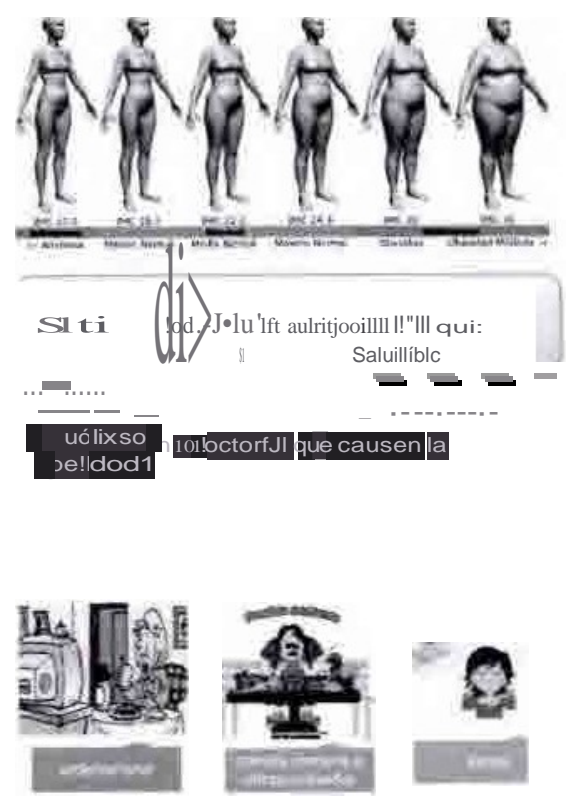

\section{- uniré}

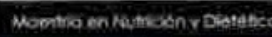

ALIMENTACIÓN PARA PERSONAS ADULTAS CON OBESIDAD

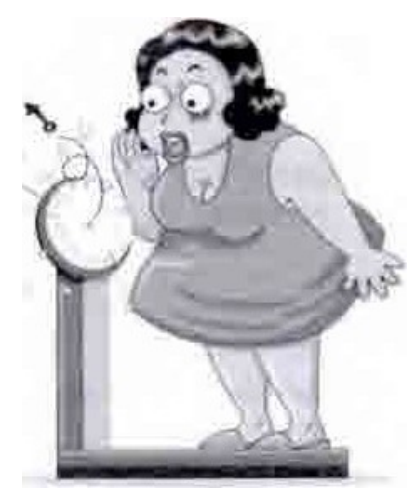

2016

\section{- \\ Cómo se trotab abe!ld ad}

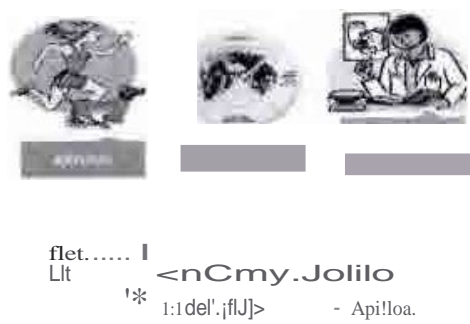

\section{Como debe comm uno persono}

con obctldoc

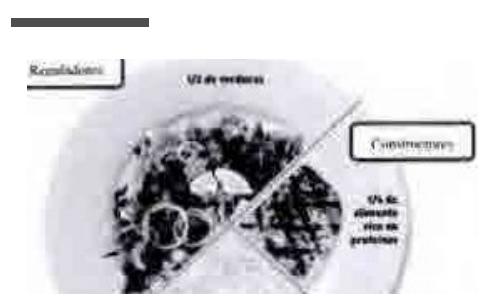



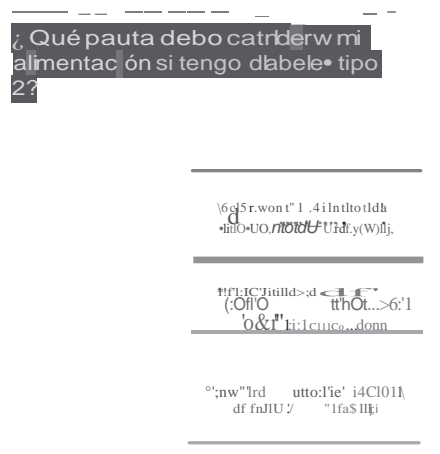

¿Cuántos minutos debo hocor eje;clclo oltengo d abetes tipo 27

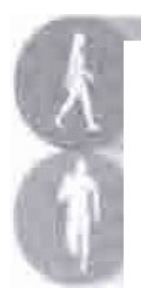

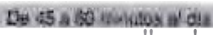

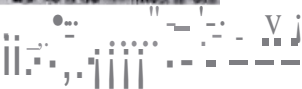

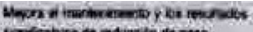

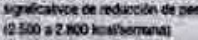

R.tt1td11l

SJ i dlahew<y; ;_llif mwCZar ho.: a

c) $\rho_{i t l}$ 'il:io deb•d vi<i!Ar -' mcdicq y

'11Uiric- !"' IW)lar lim'o.Jsili1<11qdldll;
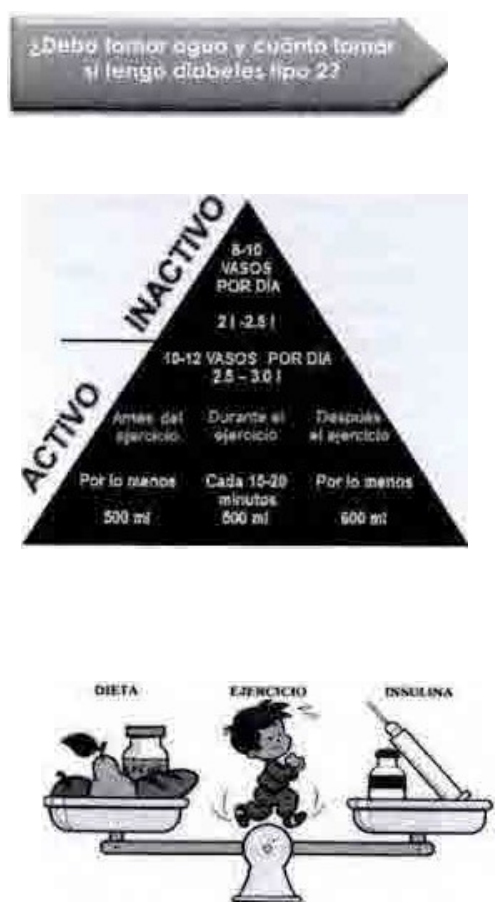

Es<;::ueld def'o\$t-godo U;|IJF
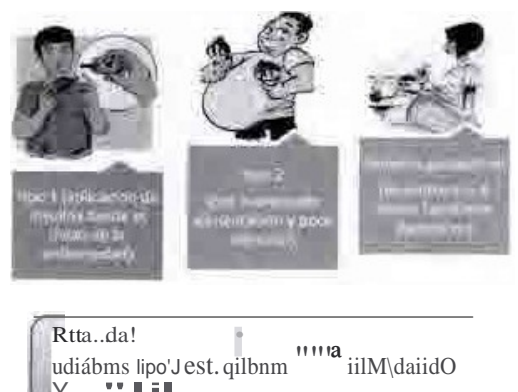

udiábms lipo'J est.
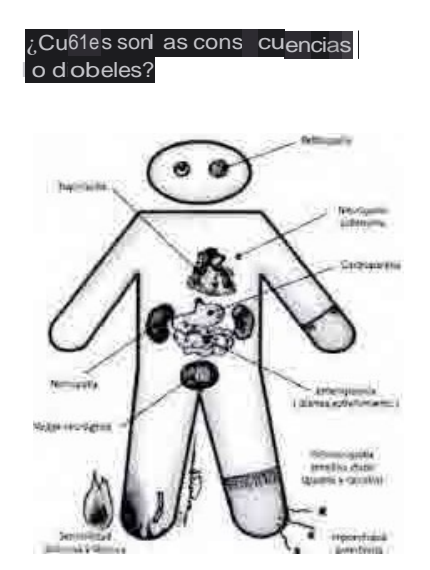

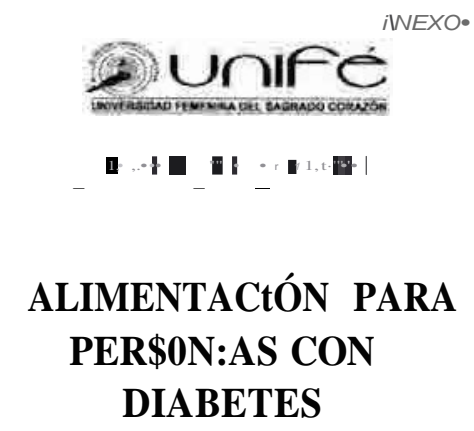

DIABETES

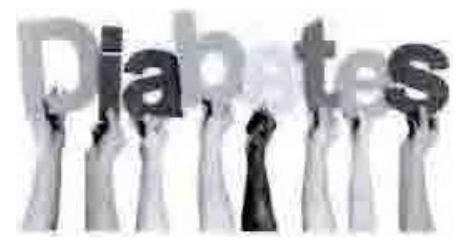

2016

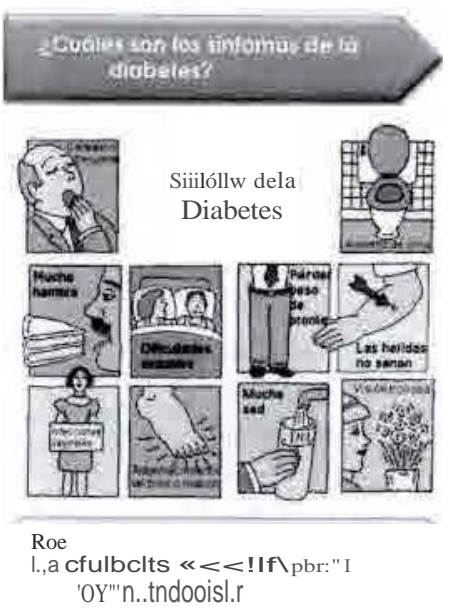

¿Cual ses ellotom enloded abet s?
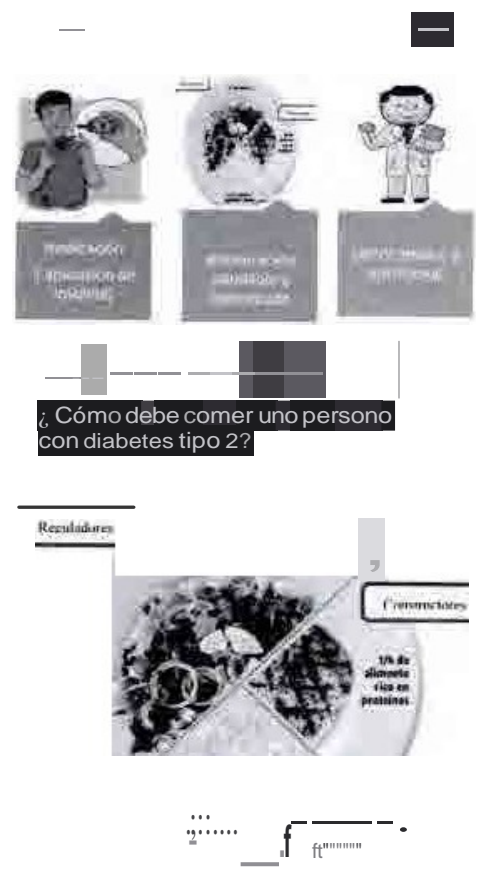

Rttllft'da!

1.jlfuilin.;.dd>e.coot e1

in\$11 núlp.ili,.,m... 
Enfem1edades CAtdiollIISCIÁlltes (ar1mioescleros1 1 o II:larto al $\mathrm{m}$ rdlo)

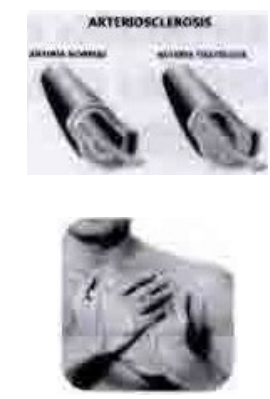

Sobrepeso y Obesidad

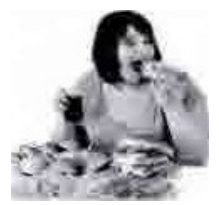

J Jeva -dlela ccmumodelfut.

Aomenla

2. RaCr \|ӥ:a

3 ya le d8・llmenl!>s degnm éj:>(.l'én140 llh ClllQrliaa,

4. .,.,..menta.Qioan1umo de-libra,@'t.,.,pecial qe $1 \mathrm{~W} 8<\mathrm{UL}$ '/ c:'.ef1l...r.g.

S. R $=$ OM-O-3,PftCadOy $e^{\mathrm{R}-二=}$
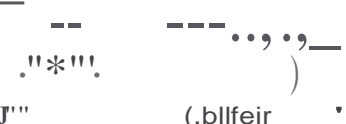

$r$ QptaJ"' 19che, fQI4MJYJo9ur

ftlluras,tabec:o yatc llhot
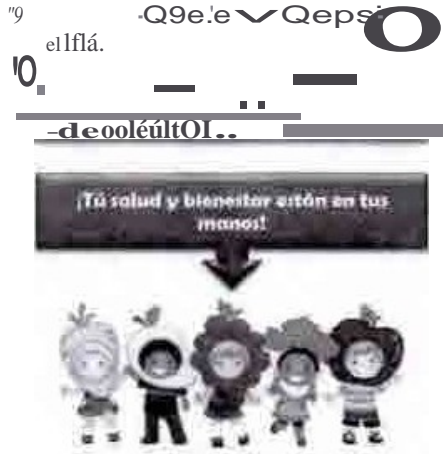

Eurcuibla de Pont progo unife
AUMENTACIÓN EN

PEBSOAASTR ON $^{2}$

ILIVADO

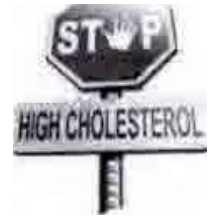

'El coJo ffH'Ol elevado en Slfng uno de/os (J'Inclp $\bullet /$ factora de riesflp cettlfovucul•r $r$-stglln •I InsOtuto del Contdin \$ru.

\section{CoMO ACTÚA ELCOLESTEROL EN NUESTRO CUBRPO}

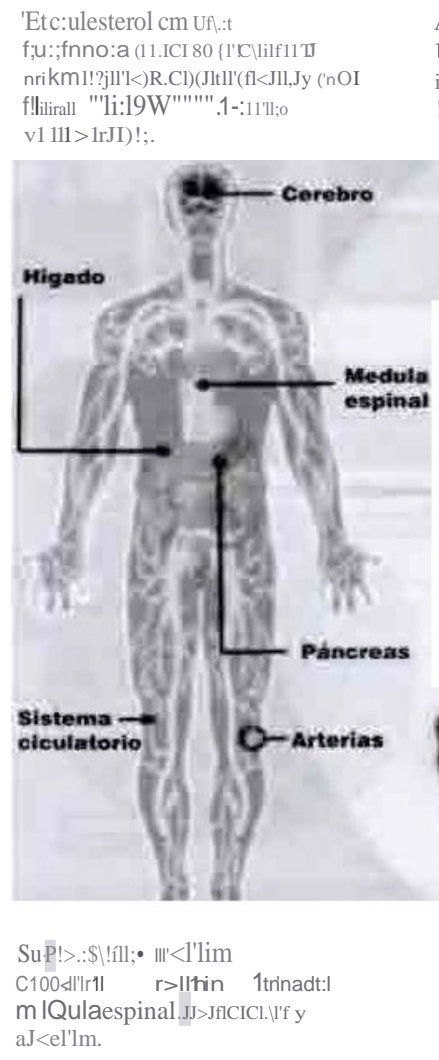

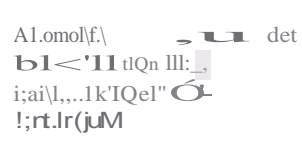

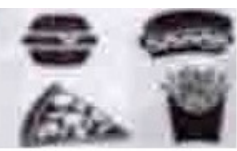

$\mathrm{E}<1$ !VJllitt.c "' Jite en Irl!ll mhililono:; $y$ j1'UM! ullu(l'MifO)

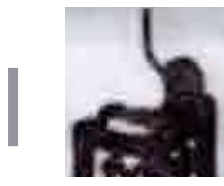

t:t dl119.tloo vi• oámen de
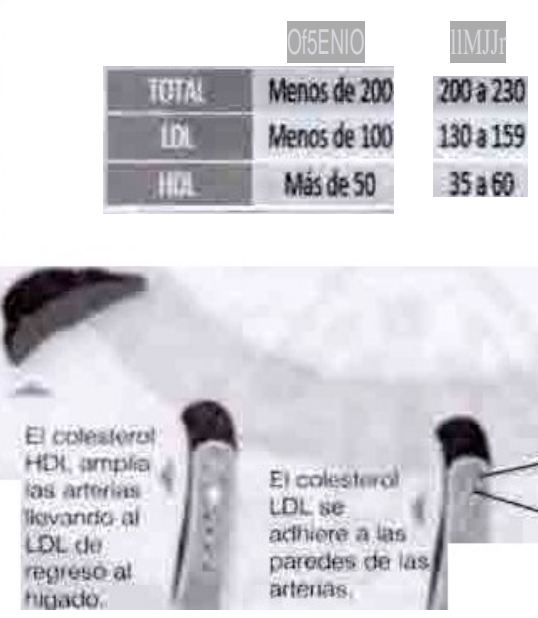

El colesterat LDL se acthere a tas paredes de las artenas.

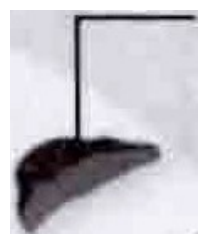

$(,, \quad--t \bullet f t|\cdot \bullet| F I I ́ s 3$ ,iffl.1\1c:ill C $>$ Jillli $>>$ JJUro et ui.;i・1l•y 1 f1N, 111(tl.um 1 co14Ju
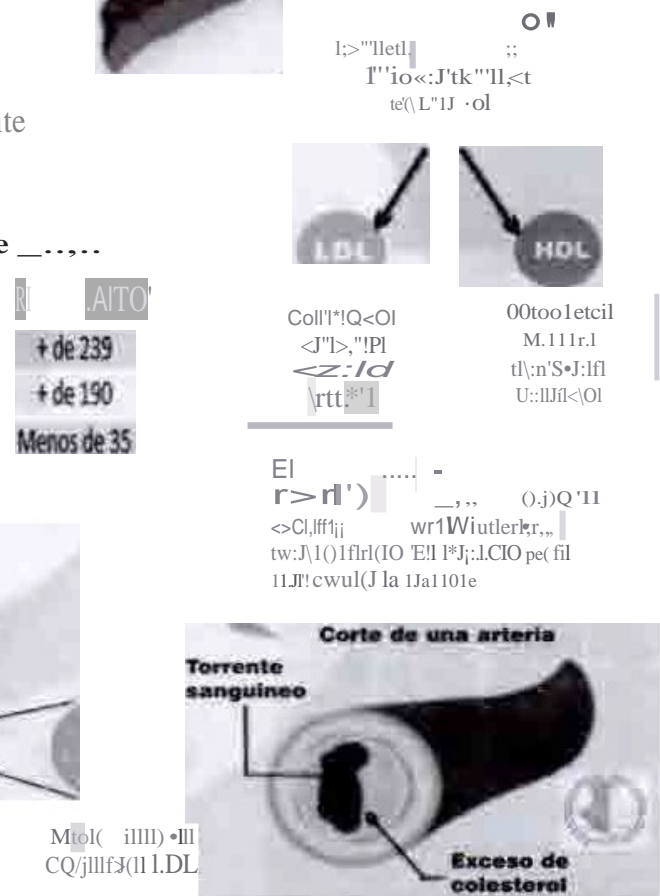
Cuáles son fas consecuencias deta Hlprt'iglicéridemia?

ethcremento de los nivelesc:Je tligOcérldos $\mathrm{pQr}$ encima de bs parámetros recoróéndados puede ocasionar:

.Enfemledades careiovascula(es

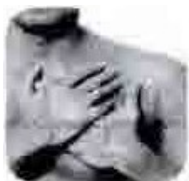

[abetes Melltus 2

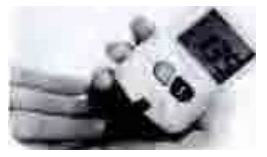

Sobrepeso y; Obltsidad

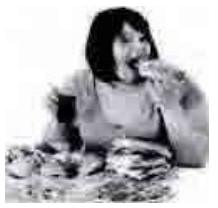

2.Q.ué son los tilglicérldos?

Forma de almacenamiento de energla que 5 e d<llJOSila en el músculo y en el téjjdo adiposo que son gradumenta lít>eradoœle acuérdo a las nece,idjdits de energh:

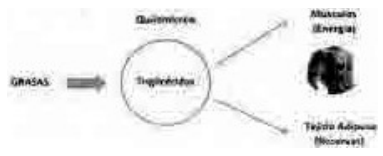

iQu son trigUcéridos altQs!

Trigl eéridos łps o hlpertrigllcéridemia es cuiindo el nivel de ItiglTcétiljos en sangre se encuentra por encima de los valores normales.

\section{ITJ TRIGUCRIDOS}

$$
\begin{aligned}
& 500, ., j_{., .,} \text {- > Muyalco } \\
& \text { 200-499 mist कु } \\
& \text { 1SO-199,.... ? lfmfte } \\
& \text { 드"1 - • • Ópúmcl }
\end{aligned}
$$

IMPORTANTE :

1. leva una diéla ballJ'cead Aumenta el oonsumo de frutas-y verduras.

2. Rlza!IC!lvidlid!laicademanera regular

3: Q rnlt•uyalahge)Ilide łnentc1s déwají Cbntetúdo <encalones.

4. Aórnenta; ;CQ!..ISUmó de fib en . ai deb.avena y atrealá inleglaltis-

Cona omega $3, \quad y$ olltlelil «111' oliva,

6: Oismliul''9ks,'QrasaS< satUtaclaS' y, 1111ns frituras;

lleffá.

T. Opta $1 \mathrm{pb}$ Cl!álas en gta!Ul-J leche, qoJMO yogur delcnlmado.

\&, el 8a.a, Irilurnfilbaco )'Jlklóllol.

Evllla'81 сомuml) $\Longleftarrow ~ 1 l$ mplea. P!!lle acter
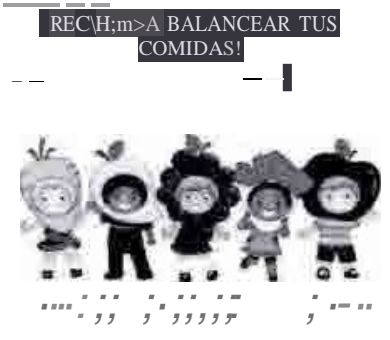

II'or q11é se presentán triglicérldos elevados .en s-angre..?

La Hipertrigllcérldemia se puede se deber a causas 9enéticas. enlerif19da<; Jes oo o l en dieta muy rlcil en azucares y grasas. el consumo excesivo de alcohol da rana de aclividad fisica

$$
\begin{gathered}
\text { ¿Qué almentos debemos tomar } \\
\text { en cuenta pata evitar la } \\
\text { preJ>encia de triglicéridos } \\
\text { elevados en sang(e? }
\end{gathered}
$$

Almertos refinadosy azúcares s mples

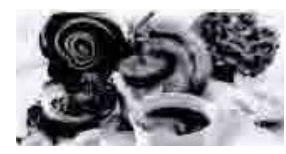

Afimentos con§o contenido degrasas

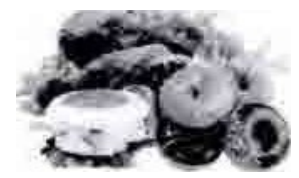

Bebidás azucarada\$ y alcohol

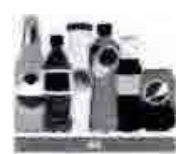

i

Mosstra er Numbán y Dieteldico

\section{AUMENTAQÓN EN \\ PERSONAS CON \\ TRIGUCERIDOS}

ALTOS

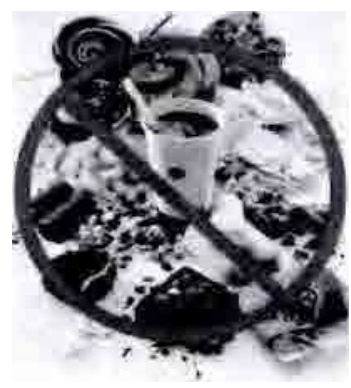

"Los triglicérido\$ efevado1t. e re/aclonprl11cfp11/menf<1 conel cons umo exceSlyo de aJirpentos refinados,. alcoholy•zúcares"...

$20 \backslash 6$ ¿cuáles SQnbs slntomas dẻs triglicéridos levados?

Aumento de a '9rasa abdom nal.

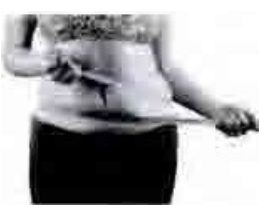

Dolores de cabeza problemas óehsomri

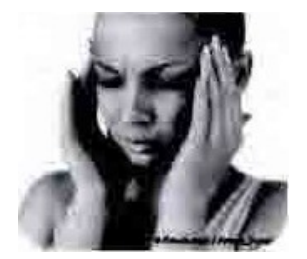

Ansiedadpoicomerdutoes.

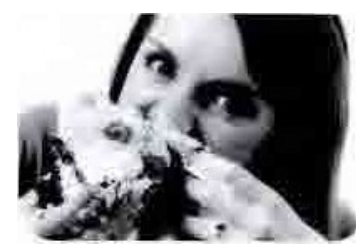




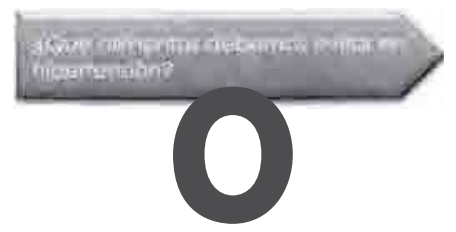

kWMOS NJW..1!.XJS OUICID)'-r.

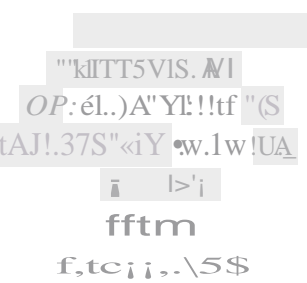

|t.-OIAS arr...II-'SIIIII'IX.'tiAI)

OCO"tLALL.'!S fif1i.NII:OOfWIAfT:Ji
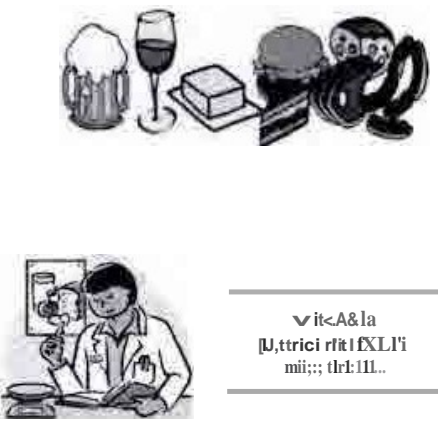

vit<,A\&la

[N,ttrici rlit | fXLI'i mii;:; tlr1:111..

\section{¿Qué <>b liperterislon arteria?}

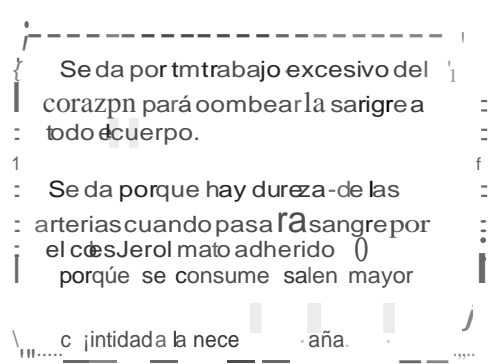

\begin{tabular}{|c|c|c|}
\hline Categoria & PA sistblica & PA diastofica \\
\hline & & \\
\hline Normal & स्रूपर & 20ब्ब \\
\hline Wormal Alta & $130-839$ & $85-89$ \\
\hline HTA Grado t leve & $740-159$ & $96-99$ \\
\hline $\begin{array}{l}\text { ATA grado } \\
\text { moderads? }\end{array}$ & $1005 \times 9$ & 100,102 \\
\hline$x=12=$ & F & STe \\
\hline
\end{tabular}
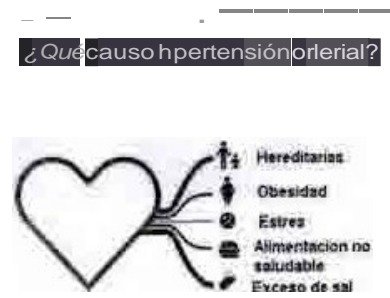
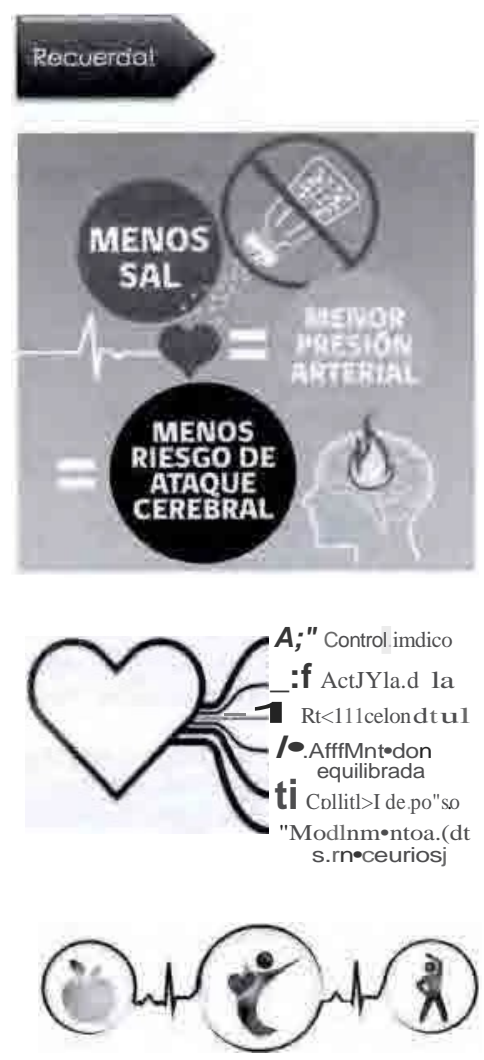

Que debe comer uoa persona con hipertensión arterial?

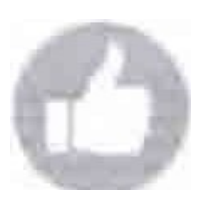

fi U A

VLIIDJ?AS 'f EC;CAS

1 IJTOSg $g_{2}$ COS UDOS O fOSIADOSSINSAL CAAAiE BIAMv

JESCAOO 11.LUL

rtsr.ADO 6!ANCO

Lt.GUM131'LS

CTREAl.fS

?.. \$' A.LIMENfOA

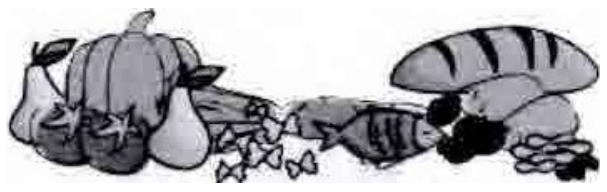

\section{uniré

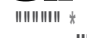 \\ wo,.}

Maestria en Nufricion y Dietefico

\section{ALIMENTACIÓN EN \\ PERSONAS CON}

HIPERTENSIÓN ARTERIAL

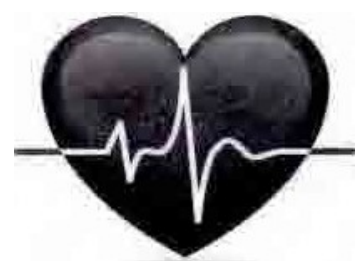

"'IVSM.V $<D$ 7'

(\}JIR'ES $<$ IJL P:N TVS 'M.ft!NOS.

016

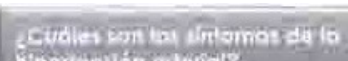 hiponotalion suterich?}
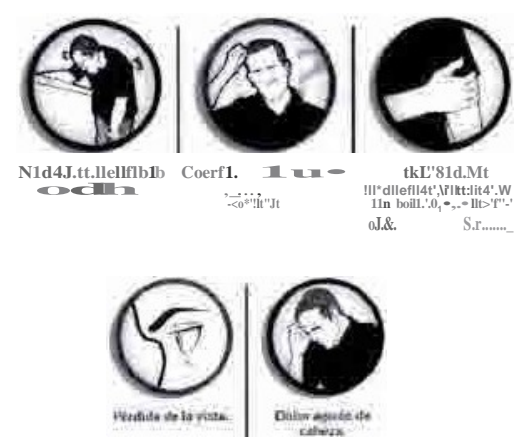

(2) de la hupentemion antental?

COMPLICACIONES PRINCIPALES DELA HIPERTENSIÓN

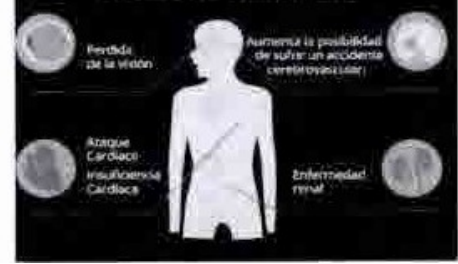




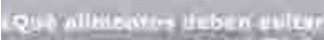 \\ uns porsone con griekrtals?}

Fru1<1s citriéas (naranja.. t6ronja pina, $i$ ie)

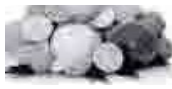

Frtturas ocomida répida

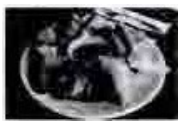

Café. té 0 bebidas con alio conten1do de calelna.

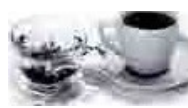

Ajly condimentos.

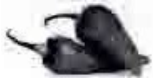

Fumar y. C01'1Sum9 de bebidas alcohólicas.
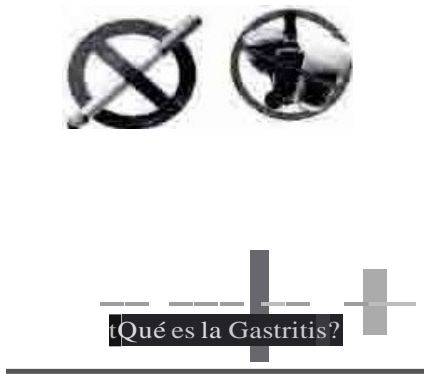

E:S la Inflamađóh de las pareces del estómago.
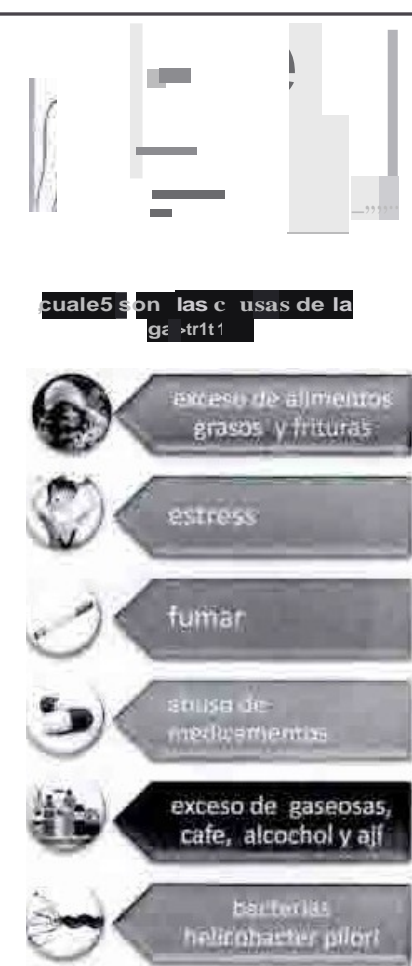

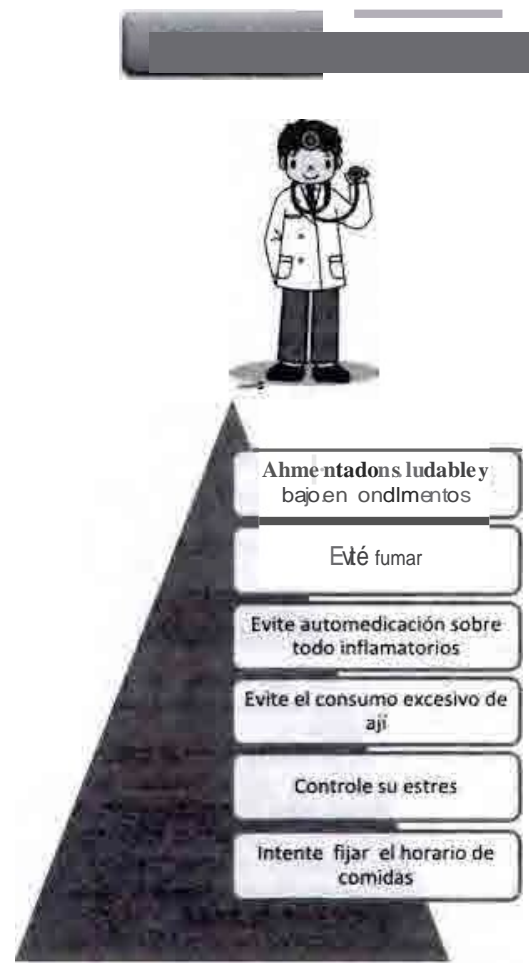

isita al méd zo. La gastritis

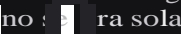

\section{lun1Pé}

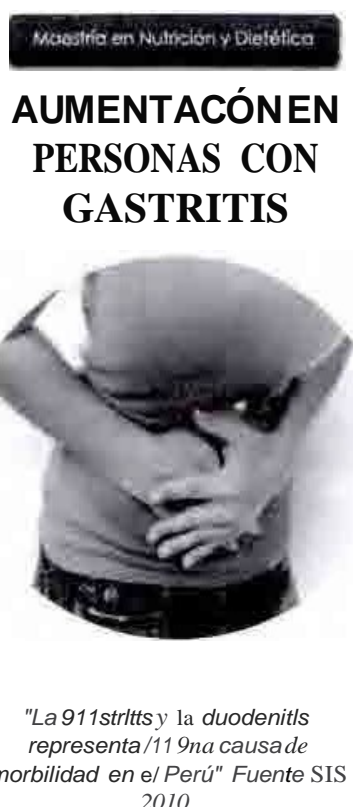

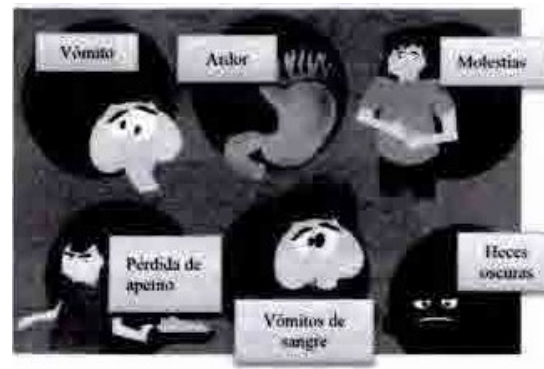

I.Cuáles son tas c onsec encias de la gas itis?

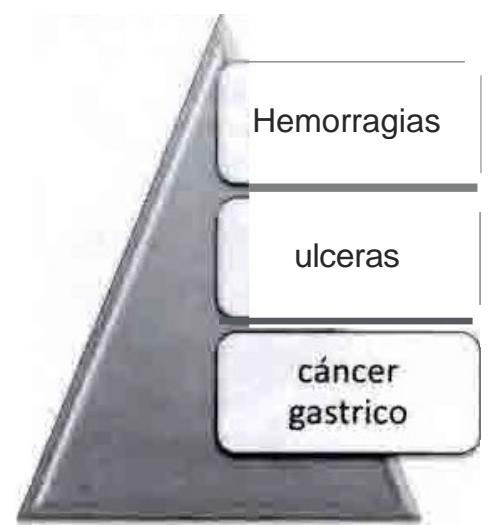

¿cómlo se de be alhne personalcon gastrittis?

Comer a ta Hpra

\section{Almpuloseconondsdo:}

Cablan.cas

def! $¡ c$.dlgestl6n.

E,Je' pe

potld.i pavit\&,ent otros.

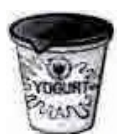

Lácteos.y

deivados descromados o

semídes.cremados.

Ejemplo;yogurt Ight

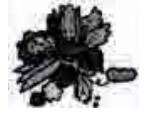

Verduras de preterenciiis cocidas.

Frutas natures como por. ejempló: papaya

jecuerd $\bullet$

AC:ud• a 1. a nuttldonküpaA Un tNlt1rliento .Personalizado y evttar c:omplkadonos... 

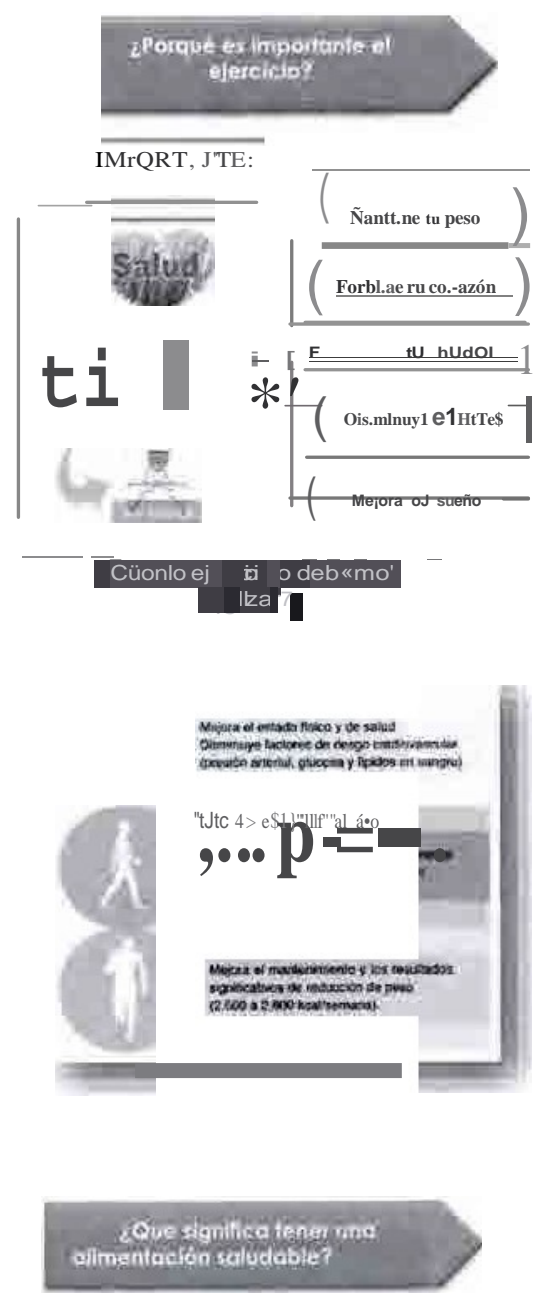

\begin{abstract}
\$nJfica saber etegr :¡11lmentos que \$nJfica saber etegir :i11mentos que
aporten todos. los nutrientes esenciales y la energia ql!e cad ${ }_{i i}$ na neceifa para manteneraesana EsIQs nutrientes es,enc;ales són: proteinas.
\end{abstract} carbohidrato, gl'á as. Vaminas;
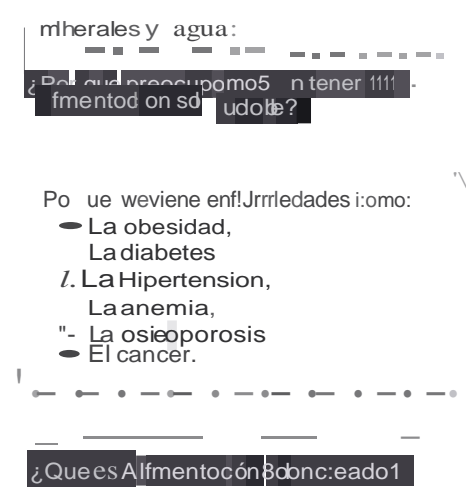

\section{¿Que es Alfmentocón Bobnc:eado1}

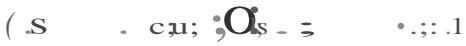

- alimentos en la ł̇nentación diaria en . t pqrciones ade<illildas ab edad.al peso, 1 l a 1alla y a la actividad isic:a

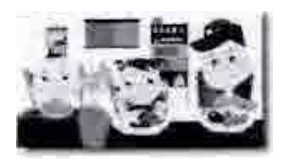

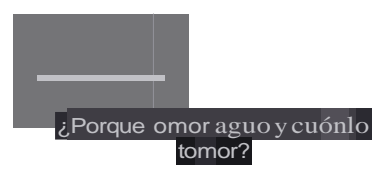

REG?IA 1ATEPER...TURACORPQRAI pAO'ÍÉGt IOS OllGANOS VITAIES

AYUDA Al CUERPO A A8SOII8ER LOS NUTRIENTES

'RREVIE.!1E ECESTIWl IENTO

fORTAlfct TU SISTEMA INr.tUN()WGICO HIORATA LA PIEL

- tNOTE UU816DEPESO EVITA IOSCAIAM:

UPONE $\mathbb{B}$ BUEN HUMOR

- REDUCE fIRIESGO ÓE PADECER CANCEA Al-COLON

TOMAR DE 6 A !IVAS05 DEAGUA DIARIO

\section{-3. Gowaerosenuvonause}

4 NTEGRE LO JNTI;GRAL A SU VIDA. $\mathrm{S}$ le<'ciOney Pf"liera alimen!OS integrads 3Yena. quinua. to stada5

REOUZCA: A CONSUMOOESAL.

Linke el uso $d$, ,.. alimentos con alto .con1rmdo de sod̈.PIJede e!Qvar pres ón. msMti'AJY.A tL CONSVMO oc RA. S Prefiera alimentos iisad. cocidos 0 . al vapor. Use \&Qeite de gfuisoly oliva, NO REEfüLíCE"LOS ACEITI!S

- IAt,UIIESUSPOS ES POR FJWTAS

Las bebibasgasificadas,jugoseo calas.por lı.191' (le fr.!Jtaś; aguarutad o agua:

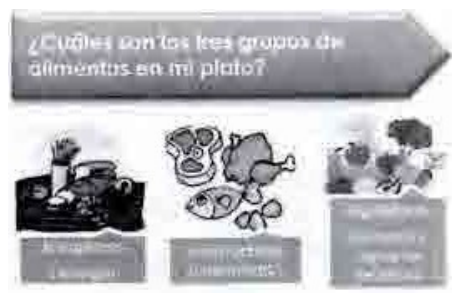

\section{Lougo logo una difuswactor} balancecda y voriado?

(i na a 1mentacion b-anceada y - - - i na a 1 mentacion banceada y variada $a-1$

1 ntercambia bs aftinentos debs 3 grupos bas cos: energeos. constructores, reguladores en cantidades :Jdecuadas para manlenerse sanos y fuerte.
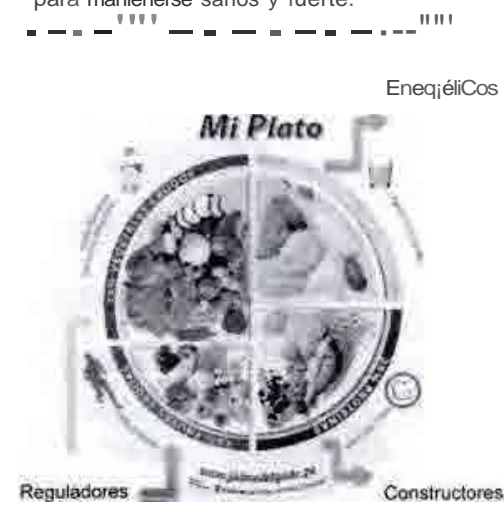

ANEX09

\section{uniré}

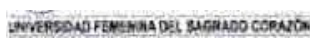

Escuela de Posgrado

Maestría Nutriclon y Oletetjc:a

An11lntomnc:iorin1 détos

\section{ALIMENTACIÓN SALUDABLE}

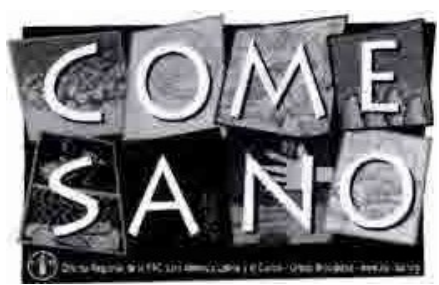

Cometes vnonece!idq pelocomeíinreiigememente

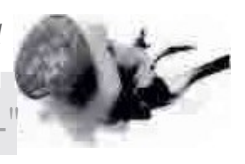

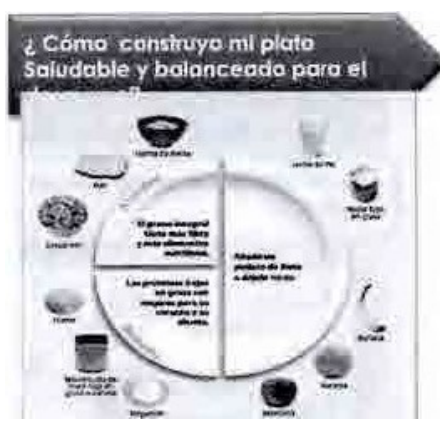
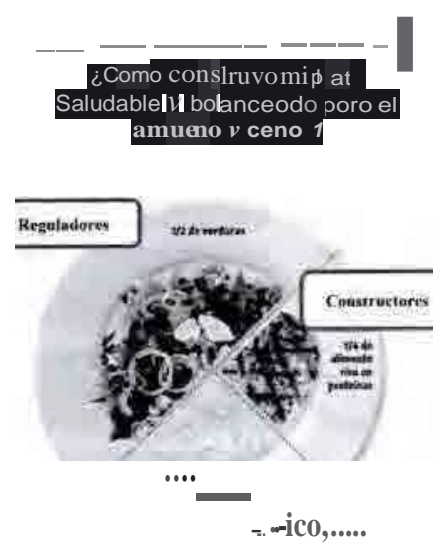

Reeuerda!

Visitar a tu nutricionista más cercana ante cualquict duda. 


\section{AUTORES}

GLORJAACOSTAA LVAREZ OE HOYLE.Abogada por la Pontificia Universidad Católica del Pení, MBA. Máster en Marketing y Dirección comercial, Auditor ISO 9001, cuenta con diversos estud ios de postgrado a nivel nacional e internacional. donde destacan estudios especializados en género como es Auditor Partici pativo en Género por la OIT, del centro ON U M UJ ERES. Líder de proyectos estratégicos de gestión en diversas organizaciones, trabaja por el ernpoderarniento femenino. Se desempeñó corno consultor internacional para temas de consumidor Microfinaociero y presidió el Comité <le atención aJ usuario en la Asociación de Microfi nanzas de Perú - ASOMIF. Actualmente se desempeña como Director ejecutivo en SAWAY PERÚ y es coordi nadora de Maestrías de Derecho de la Universidad Femeni na del Sagrado Corazón UNIFÉ.

Agradecimiento a la Srta. Oaniela Anyela Chaupis Sosa,estud ian te de Derecho, quien cooperó en la elaboración del presen te artíc ulo

LUIS ANGEL AGU íLAR MENOOZA. Director del Laboratorio de Neurociencia y Comportamiento de la Universidad Peruana Cayetano Heredia. Consultor Intern acional en NeLLrociencia. Doctor en Neurociencias y Comportamiento, Universidad Pablo de Olavide- Sevilla, España. Postdoctorado en Neurociencia, Uni versidad de Salamanca, España. Postdoctorado en Neurofisiología, Uni versidad Newcastle UK-Universidad de la Republica U ruguay. Master en Neurociencias, Universidad Intemacional de Andalucía. España. Diplomado en Docencia Universita ria, UNTFE. Estudios de pregrado en la Universidad Peruana Cayetano Heredia. Autor de 67 publicaciones entre artículos cíentifi cos. libros y capítulos de libro, Conferencista internacional en 20 paises, revisor de 4 revistas indizadas internacionales. pertenece a 14 sociedades científicas naciona les e intemacionaJes. Docente de pre y postgrados de 10 universidades del Perú.Neurocie ntífico.representante Peruano ante la lmemational Brain Research Organization y federación Lati noamericana de Sociedades de Neurociencias. Presidente de la Sociedad Para la Neurociencias del Peru, SONEP (2015-2018). CATEDRATICO DE NEUROCfENCIAS DE LA FACULTAD DE PSICOLOGIA DE LA UNIFE.

BYRON ESTUARDO A LVIZUR ES VALLEJO. Médico cirujano. Estudió en la Universidad de San Carlos de Guatema la, Centro Universitario de Occidente. Realizó una estancia certificada en fonnación en Ecografia en el Hospital Roosevelt, Ciudad de Guatemala. Así también, realízó tina estancia para el estudio de Col poscopia en el Hospital Universi tario Príncipe de Asturias, Alcalá de Henares, Madrid-España. Actualmente ejerce la especialidad en Ginecología y Obstetricia en la Uni versidad Marian o Gálvez de Guatemala. Recibió el Premio Único concedid o en el Curso lntemacional de Bioética y Bioderecho del Insti tu to de Salud Carlos !Il de España y la Uni versidad de San Carlos de Guatemala. 2004.

MAN U EL ARBOCCÓ OE LOS HEROS. Licenciado en Psicologfa. Con estudios concluidos de Maestría en Psicología en la Universidad Nacional Mayor de San Marcos.

Profesor Universitario. Docente en la Facultad de Psicología y Trabajo Social de la Universidad Inca Garcilaso de la Vega (UIGV) y en Docente en la Universidad Femenina del Sagrado Corazón (UNIFÉ). Coa Fonnación Psicoterapéutica corno Analista Transaccional Certificado en la Asociación para el Desarrollo Humano Xinergia. Segunda Formación en Logoterapia Clínica en el Centro Psicoterapéutico LOGOS con el auspicio de la Sociedad para el avance de la Psicoterapia centrada en el sentido (SAPS) de Bogotá-Colombia. Miembro del Colegio de Psicólogos del Perú.

Es autor de varios artículos de Psicología y Humanidades en revistas de la especia lidad.

Articulista del Diario Oficial El Peruano desde el año 2014.

Ponente en jornada s de Psicología, Educación y Psicoterapia. 


\section{UNIFÉ-EPG}

MA RÍA L UCÍA BAQUERIZO SEOANO. lnvestigador asociado al Laboratorio deNeurociencia y comportamiento de la Uni versidad Peruana Cayetano Heredia-UPCH. Psicóloga clínica en el Equipo de investigación y tratamiento en aspergery autismo-ETTA. B.Sc. en Psicología en la Universidad Conti nen tal. Becaria dela fntemational Brain Reserach Organization IBRO. Asistente de cátedra en universidades peruanas a nivel de pregrado en cursos de neuroanatomía y neurnpsicolog(a. He desanollado proyectos en neurociencia cognitiva, sueño y adaptación fisiológica a la altura. Temas de interés: Síndrome de asperger, Condiciones de espectro autista, Neurodesarrollo y Neurorehabi litación.

AGUSTIN CAMPOS ARENAS. Graduado y titulado como profesor de Educación Secundaria: Física y Matemática en al Pontificia Universidad Católica del Perú donde también se graduó como Doctor en Educación. Obtuvo el Master en Educación en Temple University-USA y el Ph.D. en Sistemas lnstruccionales en Florida State University. Real izó estudios en Educación No-Formal en las Universidades de Manchester -lnglaten-a y Edimburgo-Escocia.

Se desempeñó como especial ista y Director de Formación Magisterial en el Ministerio de Educación. Ha sido Jefe de Departamento, Decano de la Facultad de Educación, Vicen-ector Académico y DI.rector de la Escuela de Postgrado de la UNJFE.

Cuenta con varias publicaciones relacionadas con la educación en UNfFE y la Editorial Magisterio de Colombia. Ha hecho consultorías i nternacionales en educación en diferentes países.

ALESSANORO CAVIGLi A. Magíster en filosofia por la Pontificia Universidad Católica del Perú. Profesor de la carrera de Filosofia de la Universidad Anton io Ruiz de Montoya, profesor del Departamento de Humanidades de la Universidad Ricardo Palma y profesor de la Facultad de Derecho de la Universidad San Martín de Porres. Ha publicado diversos rutículos y ha presentado numerosas ponencias.

Jl:IONATAN ROBERTO ASTUCURJ HIDALGO: Psicólogo por universidad Continental. Con foanación en psicoterapia por el Instituto Gestal t de Li ma. Realizó l a formación en neurociencias en 'Laboratorio de Neurocien cia y comportamiento" de la Unjversidad peruana Cayetano Heredia. Hízo un Trai ning en "Neurobiología del sueño", en la Universidad de la Republica, Uruguay. Participo en "V Congreso Internacional de Investigación y Práctica Profesional en Psicología" en la Universidad de Buenos Ai res. Realizó el curso "Metodología de la investigación" de la Universidad de A lmería, España. Dirigío el "Laboratorio de Psicología experimental y de neurociencias"de la Universidad Continental, y real iza proyectos en colaboración con la UDELAR, UPCR y PUCP, en temas de Altura, utilizando técnicas de registro electrofisiológico. Habiendo publicado en revistas científicas, como la revista "Apuntes de ciencia y sociedad" de la Universidad continental. Y presentado sus trabajos en congresos de estudiantes y profesionales a nivel nacional y mundial. En la actualidad trabaja como investigador en el Laboratoriode Neurociencia y Comportamiento de la Univers idad Peruana Cayetano Heredia.

A LEXA N OER CHJ U WERN ER:Master Executive in Global Entrepreneurship en la EOl de España. Licenciado en comunicación y publicidad (UPC) coo estudi os de postgrado en marketing, neurociencia aplicada a los negocios y medios digitales en Perú (UPC, UPCH) y Aleman ia (DW-Akademie). Actualmente es director de estrategia e investigacíóo eo NEO, consultora de innovación y estrategia digital, además docente pregrado y postgrado (VPC, UP). Cuenta con un blog en Gestion.pe.

ALEX JUNIOR GON ZALES ASENCIO : Investi gador Asociado al Laboratorio de Neurociencía y Comportamiento de la Universidad Peruana Cayetano Heredia - U PCH. Psicólogo por la Universidad Peruana Cayetano Heredia. Estudios de Maestría en Neurociencias por la Universidad Peruana Cayetano Heredia. Becario de la lnternational Brain Research Organization - IBRO. Actualmente realiza labores de investi gación. coordinación y docencia uni versitaria . 
ELENA NELLY GARCÍA - L.ÓPEZ RERNA N DEZ. Doctor en Ciencias Biol6gicas por la Universidad de Alcalá. con la calificación de Sobresahente "Cum Laude" (1982). "Tones y receptores i mplicados en la modulación de la liberación de catecolaminas en la médula adrenal de gato", Licenciatura en Ciencias Biológicas. Universidad Complutense deMadrid (1 976). Gradode Licenciado en laFacultad deCie11cias Biológicas. Universidad CompJ urense de Madrid (1979). Hasta el 2016 fue profesora eméri-ta de la Universidad de Alcalf1Madrid. Los cargos académicos desempeflados fueron: SECRETARIA DEL DEPARTAMENTO de fisíología y Farmacol ogía de Ja Uajversidad de Alcalá de Henares (desde 1986 hasta 1996). SECRETARI A DE LA FACUITAD DE MEDICINA de la Universidad de Alcalá deHenares (desde 1992 hasta 2000).SUBDIRECTORA DEL DEPARTAMENTO de Fisiologia y Fannacologia dt la Universidad deAlcalá deHenares (del 28 de febrero de 1996al 8dejulio de 1997). SUBDI RECTORAGENERAL D.E PLAN fFlCACIÓN Y COORD1NACIÓN DOCENTE del lnstitulo de Salud Carlos Hl, Ministerio de Sanidad y Consumo (del 1 de septiembre de 2000 a.1 30 de agosto de 20ro). SUBDfRECTORAGENERAL DE POR M ACJÓN Y PLANIFfCACTÓN DE LA INVESTfGACLÓN SANITARJA del Instituto de Salud Carlos Jll, Miniscerio de Sanidad y Consumo (del 31 de agosto de 2003 aJ 30 de septiembre de 2004),

GU TAVO FRANCISCO GONZALES RENG IFO: Biólogo y médico end ocrinólogo, profesor principal del Departamento de Ciencias Biológicas y Fisiológicas y director del Laboratorio de Endocrinologíay Reproducción de la Facultad de Ciencias y Filosoíla de la Universi dad Peruana Cayerano Heredia. Magister en Ciencias coa mención de fisiología (1978). especialidad en endocrinología (1992), doctorado en c:iencias con mención en fis'iol ogía (1985) y doclorado en medicina (1999). Fue Director del Instituto de lnvest igaciones de la Altnra. electo en seis. oportunidades. Vicedec:"mo de la Faalta d de Ciencias y filosofia en dos oportu ojdades (1999-2002:2002-2005) y una vez electo Oecano (2005-2008). Es director del Círcalo de nvestigación en Plantas coa Efectos el,'! Sal ud. Bn los dos años de vigen:cia, elCirculo ha logrado diez Premios lladonal es e i nterm1cionđes. La demostración que la mezcla de maca negra y hojas de yacón revierten la hiperglice!Uia y la disminución eu el c011teo de espermatozoides le ba pennitido obtener una pa tente ea Estados Un idos. En los 16 años cjue el equipo del Dr. Gonzales trabaja en maca ha logrado un reconocimiento muy importao tc a nivel nacional e intenlacional. Ello se refleja en las J1Umerosas publ icaciones sobre nlaca eo revistas indexadas.. su aparición en numerosos medios masivos dé comun icación, tanto n acionales como internacionales. Esta labor se ha \risto reflejada en las exportaciones de maca que del 2001 ni 2015 se han incrementado en 30 veces. sitmdo la maca la planta nutracéu tica que más se exporta del Perú Ha asesorado 29 Lesís de bachiller/licenciatura, 17 tesis de magister y 6 de doctorado. Sus artículos han sido citad os 3540 veces y ti ene un indice $\mathrm{H}$ de acuerdo a researchgate de 35 y de scope de 27. Es autor de más de 300 artículos ciel'ltíficos y ganador de 53 premios y/o reconoci mi entos nacionales y 9 inlemaciona les. Es Acadé mico de Nú mero en la Academia Nacional de Medicina donde es Secretario Bienal y Academia Nacional de Ciencias donde es Vicepresidente.

JORGE LUIS LINA RES W EI LG: Licenciado en Publicidad. especializado en Marketing y Marketing Digital. Coordinador del Área de Neuromarkt:ting del Laboratorio de Neurociencia y Comportamiento de la Universidad Pert1ana Cayetano Heredia. Investigad or en Comportamėnto del shoppedconsumidor. empresa. sociedad. famil ia. ética y entorn o. Coafereocisra eo Tendencias e I nnovación Estratégica, uaa perspectiva neurobiológica (Vniversidad Nacional de Tumbes). lV Congreso Internacion I de Market ing (Uni versidad Co.ncinental, Huancayo). Diplomado Psicología Sodal ,Neurom nrketingy Procesos Políticos (Universidad Nacional de San.Agustín, Arequipa), Semillarfo Internacional deMarketi'ng(Uni versidad Privada del Norte, Trujillo).Promperú,Cofide, Expomarketing. Coordinador y docente del Oiplómado en Nc:uro marketing y Neurociencia aplicada a Los Negocios, U niversidad Peniana Cayetano lleredia. Coordinador de la Semana Mundial del Cerebro 20'15 Ll MA, PERÚ, por Dana Poundation (Brnin Awareness Week). Onizador de Encuentros N, Seminario de Neurociencia y Negocios. Universidad Peruana Cayelano Hered.ia. Estu dios. de postgrado en E-Marketing, Estrategias e lmplemencacián en ESAN. Especialización en Gerencia de Proyectos - PMI, en la Universidad Nacional de lngenicria UNL Profesor de Neuromarkeling en la Universidad Continental. Análisis Publicitaritl en la Universidad Nacional del Centrn, Huancayo.Profesor de Shopper Marketing, Marketing Digital,Esttategias de Comunicq ci<,)n de Marketing en el lnstiLuto San 11!,Tlacio J SJL. Profesor de Psicología de la Müsica en la facultad de Música de la Universidad de Ciencias Apl icadas (UPC). Profosor de Brand ing y Packaging, Community Manager, Funda mentos de Marketing y Publicidad en la Uni versida d César Vallejo(UCV), 


\section{UNIPÉ-EPG}

Profesor de Psicología del Consumidor, Tecnologías de la Jnfonnación y Comunicación en la Universidad Lnca Garci laso de la Vega (UJGV),

CÉSA R LU IS MALPA RTI OA PORRAS.Profesiona l Psicólogo egresado de la Universidad Peruana Cayetano Heredia. Miembro del Equipo Técnico de Rendi miento y Competencias de la Dirección General de Gestión del Desarrollo de Recursos Humanos-MINSA, que contribuye al desarrollo y logro de su visión y misión insti tucional; poniendo al servicio la formación profesional con eficacia y eficiencia, demostrando calidad. calidez y compromiso profesional en el desenvolvimiento propio de las funciones. Docente universi tario (presencial) y tutor (online) por la Uni versidad Peruana Cayetano Heredia - Universidad Pompeu Fabrá, UNTFE y ESAN, en materia de Sistemas de lnfonnación para la Gestión de las intervenciones sanitarias, Metodología de Investigación y Recursos Humanos. Dominio probado del campo psicométrico (construcción de instrumentos), Base de Datos (Estructura y Admi nistración) con el uso de di versos programas estadísticos aplicados a la Investigación Científi ca (SPSS, STATA, Epilnfo y Eview). Profesional con experticia en evaluaciones y entrenamiento en Gestión de Recursos Humanos por Competencias según políticas rectoras de SERVIR (Reclutamiento. Selección y Evaluación del Desempeño, entre otros). Experiencia Admi nistrativa y Operativa en Salud (Programa Nacional SAMU, Di rección de Salud !V-Lima Este, Ministerio Público, EsSalud e Instituto Nacional de Salud Mental "H. Delgado-H. Noguchi..).

CI NT H YA VANESSA M INAYA LESCANO. Tiene experiencia en el área de Biologla General. con énfasis en Neurociencias. Actualmente es resista y practicante del Laboratorio de Neurociencia y Comportamiento de la Uni versidad Peruana Cayetano Heredia. Becaria de la Intematiooal Socjety for Neurochemistry, asistió al 3rd ISN Laiin American School of Advanced Neurocbemistry desan-ollado en Montevideo-U mguay, 2014. Asistió al Vi Cu rso de Neurociencias (Teórico - Práctico) en la Universidad Federal Río Grande do Sul - Brasil, organizado por la lntemational Brain Research Organization, 2015.

CARM EN ESTELA MONTENEGRO SERKOVIC. Directora de la Carrera de Psicología de la Universidad Peruana Cayetano Heredia. Coordinadora de la Maestría en Psicología Clínica con Mención en Niños y Adolescentes. Doctora en Ciencias con Mención en Psicología (Universidad Peruana Cayetano Heredia, 1997, Lima). Magíster en Ciencias con Mención eo Psicología (Universidad Peruana Cayetano Hered ia, 1988, Lima). Licenciada en Psicología (Uni versidad Ricardo Palma, 1980, Lima). Bachiller en Psicología (Universidad Ricardo Palma, 1978, Lima). Autor de más de 13 publicaciones entre artículos científicos, libros y capítulos de libros. Profesional reconocida en la Red Nacional de evaluadores Rnev Perú (Afiliado a la RlEV:Red Internacional de Evaluadores) (de 2010-2013). Miembro del Consejo Directivo de la Red Nacional de Evaluadores RNEV Perú: Vocal de Asuntos Técnico Evaluativos de Educación Superior.2010-2012. Miembro del equipo para la creación de la Red Nacional de Eva luadores del Pen'.I. Octubre 2009. Miembro del Comité Directivo de la Asociación Civil Tiberiades (ONG / de servicio social a la Infancia - Proyectos Huaycán). Miembro del Colegio de Psicólogos del Perú.

.JESSIE STHE YCY HELOY ORE DE TOMAS: Egresada de Administración de Negocios internacionales de la Universidad San Martín de Po1Tes.

HUGO SA LAZAR JA U REG U J : Doctor en Salud Pública por la Universidad Peruana Cayetano Heredia. Master en Salud Pública por la Universidad de Alabama-Birminghan.Actualmente Decano de la Facultad de Psicología y Di rector de Postgrado de la Facultad de Psicología en la VPCH. Ha sido Condecorado en el Grado de Caballero 2012 y Grado de Gran Oficial 2013 por la U PCH. Distinción Doctor Honoris Causa por la Universidad Privada Antonio Guillermo U1Telo, 2013. Reconoci miento en el área de Psicología Clínica y de la Salud 2013. por el Colegio de Psicólogos del Perú. 
GERALDIN E SALAZA R VARGAS. Psicóloga , de la Universidad Peruana Cayetano Hered ia, Maestro en Psicología con mención en Neuropsicología de Ja UPCH, estudios de Doctorado en Psicología por la UPCH en Pre y Post Grado, Docente de PosGrado en Ja Universidad ESAN. Profesor Honorífico del Lnstituto de Estudios Psicológicos Madrid - España. Ganadora del Premio Nacional en el Área Social Comunitaria 2015, Reconocimiento otorgado por el Colegio de Psicólogos del Perú. Miembro de la Sociedad Para la Neurociencia del Perú desde el 2015. Miembro del Di rectorio Nacional de I nvestigadores e I nnovadores, DINA, creado por el Consejo Nacional de Ciencia, Tecnología e I nnovación Tecnológica(CONCYTEC).

Sl LV IA A NG ÉLICA SA LI NAS MEDINA: Nutricionista Dietista CNP 1156.Licenciado en Nutrición Humana por la U ruversidad Nacional San Agustín de Arequipa, Magíster Scientiae en Nutrición por la Universidad Nacional Agraria La Molina y Doctora en Salud Pública por la Universidad Nacional Federico Villareal. Cuenta con una pasantía en Soporte Nutricional Artificial realizado en el Hospital Edgardo Rebagliati Martins. Docente en el ni vel de pre grado y post grado en la Universidad Femenina del Sagrado Corazón. Coordinadora de la Maestría en Nutrición y Dietética y del Diplomado en Nutrición Aplicado a las Ciencias del Deporte desarrollados en la Unjversidad Femeni na del Sagrado Corazón, durante el periodo de octubre del 2007 a marzo del 2016. Miembro del Consejo Asesor del Centro de Investigación UNIFE del 2010 al 2015. Miembro del Comité de Arbitraje de las revistas Indexadas: CONSENSUS, ALETHEIA e INVESTIGARE publicadas en la Universidad Femeaina del Sagrado Corazón del 2012 al 2016. Expedicionaria a la Estación Antártica Machu Picchu en la Expedición ANTAR XXIU. Enero a febrero del 2015.

ROSSANA STODDART LANDÁ ZURI : Executive Associate ia Change Management - Cbange Management unit (Telefónica Perú). Marketing Analyst- Business Management unit (Telefónica Perú). Worthmore Brands lntern (Backus). Credit and Collection latern (Kraft Foods). Diplomado Internacional en Nenromarketing y Neurociencia aplicada a los negocios (UPCH). Uni versidad ESAN - Master's Degree, Marketing. Universidad de Lima - Bachelor's degree in Business Administration 


\section{Guía paraAutores}

La revista de 1á Escuela de Postgrado de la tJNIPÉ presen ta contt'ibu.c10nes de diversos autores que envfnn sus al'ticulos, que una vez revisados por pares, son publicados.

Tipo de Artículos

La revista de la Escuela de Posrgrado de fa ưNTFÉ pu bltca, fundame.nralrnen re. llrtícnlos sobre Educación, Derecho y Nu trición. Estos son de los ripo.s siguientes:

rl. De investigación empírica. Estudios de campo, rcaljzad os de acuerd o a n1ctodologias de investiga.e.ion propias a la natura leza del tema. Se \{ \{justan al Ggor y exigencias de la i mrestigación.

b. De Síntesis (integrativas). Estudios de revisión del c:stado de la cuestión en alguno de los ámbitos de interés de la revista antes señalados.

c. De propuestas teóricas y/o avances metodológicos que sean sugerentes p::tra los pmfesionrue.; e investigadores en las áreas de ioterés de la revisrn.

- Caratteris.ticas dese.a.bles del articulo remitido

Todo rnanuscdto remi tido debe cumplir los siguientes estáfldru:es:

I. Originalidad, el aporte ha de ser mralmeme inédim, no publicado rodo o parte en pubJicadón alguna, excepto casos muy justi6cados.

4. Consistencia metodológica, representada por el U:ie:> de métodos y técnica de investigación YáHdos.

3. Significación del tema, dada por el aporre que brindan al área de interés.

4. Configuración ética, respetuoso coa el contexto y’personas participantes. Se el'Í.tarán sesgos de todo tipo.

5. Estilo de redacción. claro, conciso y orden ado; se el'itarán jergas persona.le y epresioncs no acadénll(as.

6. Organiza'Ción en basea. rítuk,s y subtítulos. 1.os tinu <,S van y en negrita y los subtítulos en cursíva. En el interior del texto no se utifüadn negri tas, 11.í subrarado.

- Aspectos for males de los a rriculus

Los 11ráculos enúados para pobUcación deben coorene.r lo siguientes e.lem<.> nto :

a. Identificación del (los) antor (es): N9mbre completo, :1filiación aca<lémlca (institución en la que trabaja), localidad, dirección < le contacto (tncluyendo la clecrrónica)_

b. Título del artículo: represcm:a:ávo del contenido, ordenado, claro y conciso. En maytiscuJ.a, negrita, i.nterlinea:do sencillo.

c. Refomen: Texto bre,re Qrnsta 150 palabras) de descripción de objetivos, método y principles resultados o conclusiones. Escrito en interlineado encifü..>.

d. Palabras claYe (ke)rwords): Hasta ul)a docena de t ntünos descriptil•os, que habrán de. ajustarse e.n la medida de lo posible a algún tesauro internacJonal (preferen temente ERJ C).

e. Texto dd aróculo: según el cipo de artículo:

Esrudms empíricos: c $<>$ mpi:ende los aparrados siguientes:

lnrroducción:incluye el problema en estQclio, i;ignificatividad los objetivos, el marco teóriC(l y antecedentes. Van c.łc mos seguidos Irno comó elementos separados.

- Ilfétodo: inclurc el rtrvd y diseño de .investigación ${ }_{1}$ población, nmestra ${ }_{1}$ i nstrumentos y materl::r.les.Puede presentarse en subtítulos. 
- Resultados: incluye presentación de resultados y la lecrn.ra de datos.

- Discusión: se discuten (analizan, interpretan, explican) los resultados encontrados a la luz de la teoría sustantiva y de los antecedentes presentados.

- Conclusiones: listado de los hallazgos encontrados con relación a cada una de las hipótesis (sub hjpótesis) o preguntas formuladas en el estudio.

- Citas y Referencias: presentadas de acuerdo a las especificaciones de la APA (American Psicological Association) o del Estilo Vancouver

Estudios de síntesis )' estudios teóricos: Ensayos que revisan los avances en un campo de in terés. Comprende los apartados siguientes:

- Introducción: incluye el tema de desarrollo, la importancia del estudio, los objetivos y antecedentes.

- Desarrollo: presentado según estructura propia.

- Conclusiones: hallazgos a los que sellega como resultad o de la investigación .

- Citas y Referencias: según APA o Vancouver

\section{- Aspectos Técnicos}

Las siguientes consideraciones deben tenerse en cuenta

a) Papel tamaño A4

b) Letra: Times New Roman, tamaño 12

c) Interlineado: 1.5

d) Númeto de páginas: entre 10 y 16

e) Procesador de texto: Word 2007 o más actualizado

.f) Tablas y Figuras: identificadas con número, título y ubicación.

Compatible con el programa de Word usado.

g) Justificación: tanto a la derecha como a la izquierda

h) Citas: debeincluir la siguiente información,

- No textuales: autor (es) y año.

- Textuales: autor (es), año y página.

i) Tablas y gráficos: deben estar numerados correlativamente y con el título o leyenda respectiva.

j) Referencias: se escribe en orden alfabético y de acuerdo a la estructura siguiente:

-Libros: Autor (es) lApellido, Nombrel, año de publicación entre paréotesjs, títulos del libro en cursiva, lugar (ciudad) de edición, editorial.

-Revistas: J\utot (es) lApellido, Nom bre), año de pubJjcación entre paréntesis, título del artículo entre comillas, nombre de la revista en cursiva, número del volumen, número de la revista cuando exista, lugar (cudad) de edición, páginas (inicio - final) del artículo dentro de la revista.

-Recursos electrónicos: Similar a libros o revistas incluyéndose, además entre corchetes la palabra On -Line. A continuación, añadir: Disponible en (incluir la dirección electrónica).

- En el caso de Nutrición y Derecho los artículos seguirán los modelos Vancouver e ISO, respecúvamente.

- Aspectos Administrativos

a) Revisión de artículos: los anículos serán recepcionadas en el correo siguiente:

postgrad@unife.edu.pe

o directamente en la dirección (posrnl): 


\section{UNIFÉ-EPG}

\section{ESCUELA DE POSTGRADO}

\section{Universidad Femenina del Sagrado Corazón -UNIFÉ}

A $\$.Los Frutales 954 Urb. Santa Magdalena Sofía - LaMolina

b) Constancia de Originalidad: los autores deberán acompañar a su artículo una carta, dirigida a la Escuela de Postgrado en la cual hace constar que el artículo es ORJGTNAL en su totalidad y que no está en proceso de evaluación para ser publicado en otra revista.

c) Derecho de autor: Los autores deben indicar, mediante carta, que trasfieren el derecho de publicar, distribuir y divulgar su producción a través de la revista de postgrado. Sin embargo, conservan la propiedad intelectual de su obra.

Nota: b) y c) están en la misma carta, cuyo formato es entregado por la Escuel a de Postgrado]

d) La responsabilidad del contenido, audjtoria y originalidad es exclusiva de los autores.

e) Revisión de los artículos: Jos artículos recibidos serán sometidos a revisión en dos instancias; e.1 Comité Editorial: podrá rechazar aquellos artículos que no se relacionen con las características de la revista.

e.2 Pares: los artículos admitidos por el Cocnité Editorial será enviados a dos jueces o árbitros para su revisión, según esquema o formulario aprobado por la Escuela. De acuerdo a los informes de los árbitros se tomará la decisión: (1) publicación sin cambios o con mejoras solicitadas por los pares; (2) no publicación. En ambos casos se informará a los autores Ja decisión.

\section{- Aspectos Finales}

a. Gratuidad de los aportes: Los artículos publicados no serán recompensados económicamente. Se consideran aportes profesionales al mundo académico.

b. Numeración: Los artículos remitidos deben ser numerados desde la primera hasta la úl tima página.

c. Corrección de estilo: Los artículos serán revisados por un(a) especialista para analizar las correcciones de estilo, si los hubiere.

d. Datos de autores: En la publicación de los artículos aparecerán solo el nombre y apeUidos y la aiiliación académica de los aurores.

e. Periodicidad: La revista de Ja Escuela de Postgrado tiene un carácter anual. 
NI VERSIDAD FElfENIN^ DEL SAGRADO CORAZÓN

ESCUEl \DE POSGRJ DO

FICHA PARA LA REVISIÓN DE ARTÍCULOS E.jIIV.ADOS PARAPUBLICAR

ARTÍCULO:

REVISOR:

\begin{tabular}{|c|c|c|}
\hline & $\mathrm{Si}$ & No \\
\hline ¿El tema del articulo t!s rdevame para est.a Reism? & & \\
\hline $\begin{array}{l}\text { ¿Esuna contribución nueva einreresante? } \\
\text { (Para los artículos Je re' sif }>\text { n esto oo será aplicable necesariamente) }\end{array}$ & & \\
\hline ¿Existe coherencia ena:e laconclusiones V los daros einformaóó o del artículo? & & \\
\hline
\end{tabular}

- Si la respnesta a l'Ul1ndelnspuntos antetioresesnegativa,por favorargumentedlm111entesus motivosagui.

\begin{tabular}{|l|}
\hline \\
\hline 2 \\
\hline \\
\hline \\
\hline
\end{tabular}

- Si b respuesta nlos tres punros anteriores es positiva, por tmøor continúe con lns siguientes.

\begin{tabular}{|l|l|l|l|l|l|}
\hline & $\begin{array}{c}\text { Complcro } \\
\text {-mcnn: }\end{array}$ & $\begin{array}{c}\text { Mi11 } \\
\text { B1cn }\end{array}$ & fü n & Poco & N!llla \\
\hline ;Etítulo refleja da.ramcnte el rema? & & & & & \\
\hline $\begin{array}{l}\text { ¿El resumen/abstract da a conocer en forma sumaria el que, el } \\
\text { cón10 y los resultados }\end{array}$ & & & & & \\
\hline ¿Las palabras claves son adecuadas? & & & & & \\
\hline,$;$ El articuloestá escrito claramente parala audiencia dela re'isra? & & & & & \\
\hline $\begin{array}{l}\text { :Las citas y referencias son adecuadas? ¿Se presenta de acuerdo con } \\
\text { la guia de aucores? ¿Son acruales? }\end{array}$ & & & & & \\
\hline
\end{tabular}




\begin{tabular}{|l|l|l|l|l|l|}
\hline Sólo p;u:aartículos empíricos & $\begin{array}{c}\text { Complcm } \\
\text {-mente }\end{array}$ & $\begin{array}{c}\text { illuy } \\
\text { lkn }\end{array}$ & Bi<:n & Poco & Nncln \\
\hline $\begin{array}{l}\text { ¿La organización del articulo se ajusta a Ja estrucrura de redacción } \\
\text { propuesta? }\end{array}$ & & & & & \\
\hline $\begin{array}{l}\text { ¿La descripción e información del método ydelos materiales son } \\
\text { adecuadas? }\end{array}$ & & & & & \\
\hline ¿Los result.'ldos se presentan claramente? & & & & & \\
\hline ¿La discusión y las conclusiones son consistentes con los resul tados? & & & & & \\
\hline
\end{tabular}

Otros comcniarios adicionales

Firma

Fecha

ACA/ad1.

Adaptación de RelieYe... Rensta Electrórúca deInvestigación y Evaluación Educativa 